\title{
Symplectic Fixed Points and Holomorphic Spheres
}

\author{
Andreas Floer \\ Courant Institute, 251 Mercer Street, New York, NY 10012, USA
}

\begin{abstract}
Let $P$ be a symplectic manifold whose symplectic form, integrated over the spheres in $P$, is proportional to its first Chern class. On the loop space of $P$, we consider the variational theory of the symplectic action function perturbed by a Hamiltonian term. In particular, we associate to each isolated invariant set of its gradient flow an Abelian group with a cyclic grading. It is shown to have properties similar to the homology of the Conley index in locally compact spaces. As an application, we show that if the fixed point set of an exact diffeomorphism on $P$ is nondegenerate, then it satisfies the Morse inequalities on $P$. We also discuss fixed point estimates for general exact diffeomorphisms.
\end{abstract}

\section{Contents}

1. Introduction . . . . . . . . . . . . . . . . . . . . 575

a. Hamiltonian Equations and the Arnold Conjecture . . . . . 575

b. The Abstract Conley Index . . . . . . . . . . . . . . 580

c. Cup Products. . . . . . . . . . . . . . . . . . . . . . . . . . . 588

2. Manifolds of Trajectories . . . . . . . . . . . . . . . . . . 593

a. Analytic Setup . . . . . . . . . . . . . . . . . . 593

b. Fredholm Theory . . . . . . . . . . . . . . . . 594

c. Transversality. . . . . . . . . . . . . . . . . . . . . . . . . 596

d. Transitivity . . . . . . . . . . . . . . . . . . . . 597

e. Orientation . . . . . . . . . . . . . . . . . . . . . . . . . . . . 600

3. Compactness . . . . . . . . . . . . . . . . . . . . 602

a. Local Convergence . . . . . . . . . . . . . . . . . . 602

b. Global Convergence . . . . . . . . . . . . . . . . 604

\section{Introduction}

1a. Hamiltonian Systems and the Arnold Conjecture. A symplectic structure on a smooth manifold $P$ is a closed 2 -form $\omega$ which for each $x \in P$ defines a nondegenerate bilinear form on $T_{x} P$. Due to the latter condition, $\omega$ provides a $1-1$ correspondence 
between vector fields $X$ and 1 -forms $\alpha$ on $P$ by $\alpha=\omega(X, \cdot)$. Then closedness of $\omega$ implies that $X$ preserves the symplectic structure if and only if $\alpha$ is closed. In this case, $X$ is called symplectic. Moreover, it is called exact if $\alpha=d H$ for some function $H$ on $P$.

We are interested in the dynamics associated with a (time dependent) exact vector field on $P$. Therefore let $H(t, x)=H_{t}(x)$ be a smooth real valued function on $\mathbb{R} \times P$ and let $X_{H, t}$ be defined by $\omega\left(X_{H, t}\right)=d H_{t}$. Then the ordinary differential equation

$$
\frac{d x(\tau)}{d t}=X_{H, t}(x(t))
$$

is called the Hamiltonian equation associated with the "Hamiltonian" $H$. It defines a family $\psi_{H, t}$ of diffeomorphisms of $P$ such that $x(t)=\psi_{H, t}(x)$ solves (1) for every $x \in P$. The set

$$
\mathscr{D}=\left\{\psi_{H, 1} \mid H \in C^{\infty}(\mathbb{R} \times P, \mathbb{R})\right\}
$$

of all diffeomorphisms arising in this way is called the set of exact diffeomorphisms. It is proved in [B] to be the commutator subgroup of the group of diffeomorphisms $\psi$ satisfying $\psi^{*} \omega=\omega$, and to be a proper subgroup unless $H^{1}(P, \mathbb{R})=0$. Considered as dynamical systems, exact diffeomorphisms should have interesting special properties. At present, most research concentrates around the simplest object of dynamical theory, the fixed point set of $\psi \in \mathscr{D}$. Here, it was conjectured by V. I. Arnold that the Lefschetz fixed point theory, which applies to general diffeomorphisms, should be replaced by a Morse-type theory. This is obviously the case if the Hamiltonian $H$ is time independent, since then every critical point of $H$ is a fixed point of $\psi_{H, t}$ for any $t \in \mathbb{R}$. Moreover it was proved in [A1] and [W1] that every exact diffeomorphism which is $C^{1}$ small has at least as many fixed points as a smooth function on $P$ has critical points. The question was raised in [A2] (see also [A1] for the case of the torus $P=T^{2 n}$ ) whether this is a purely perturbation result, or holds for more general exact diffeomorphisms. In the general case, it does not seem possible to actually reduce the problem to a function on $P$, so that the statement has to be specified. We will restrict ourselves here to the following homological estimates: If the fixed point set $F(\psi)$ of $\psi$ is nondegenerate, then we say that it satisfies the Morse inequalities with respect to a ring $R$ if there exists a homomorphism $\partial: F_{*} \rightarrow F_{*}$ on the free $R$-module over $F(\psi)$ with $\partial \partial=0$ and ker $\partial / \operatorname{Im} \partial=H_{*}(P, R)$. For a general $\psi$, we say that $F(\psi)$ satisfies the cuplength estimate with respect to $R$ if its cardinality is larger than the cuplength $\operatorname{cl}(P, R)$, which is the maximal integer $k$ such that there exist classes $\alpha_{1} \cdots \alpha_{k-1} \in H^{*}(P, R)$ of positive dimension with $\alpha_{1} \cup \cdots \cup \alpha_{k-1} \neq 0$.

We want to prove statements of this type under the following topological assumption on $(P, \omega)$ : Note that $\omega$ defines not only a (DeRham-) cohomology class $[\omega] \in H^{2}(P, \mathbb{R})$ but also a class $c_{1} \in H^{2}(P, \mathbb{Z})$ as the unique first Chern class of $T P$ with respect to a complex structure "compatible" with $\omega$ (see Sect. 1b). Consider the resulting homomorphisms

$$
I_{c}, I_{\omega}: \pi_{2}(P) \rightarrow \mathbb{R} .
$$


Theorem 1. Let $(P, \omega)$ be a compact symplectic manifold. If $I_{\omega}$ and $I_{c}$ are proportional, then the fixed point set of every exact diffeomorphism of $(P, \omega)$ satisfies the Morse inequalities with respect to any coefficient ring whenever it is nondegenerate. Moreover, if $I_{\omega}=I_{c}=0$, then the fixed point set of any exact diffeomorphism of $(P, \omega)$ satisfies the cuplength-estimate with respect to any coefficient ring.

We will call $(P, \omega)$ monotone if it satisfies the hypothesis of Theorem 1. For example, the symplectic product $S^{2} \times \cdots \times S^{2}$ of 2 -spheres is monotone if and only if each factor has the same volume. At the end of Sect. 1b, we will discuss the significance of this hypothesis and the technical problems arising in the general case.

Our methods do not yield cuplength estimates for $F(\psi)$ for general monotone manifolds. (We tend to believe that there are more than technical reasons for this.) However, they do provide a method to estimate $F(\psi)$ in certain cases (see below and Sect. 1c). An example of the type of result obtained is the following extension of a result by Fortune [Fo]:

Theorem 2. For any $n>1$, consider on $\mathbb{C} P^{n-1}$ the standard symplectic structure $\omega$ such that $\omega[u]=n$ for a generator $u$ of $\mathrm{H}_{2}\left(\mathbb{C} P^{n-1}\right)$. Then the number of fixed points of a symplectic diffeomorphism on a product

$$
P=\underset{i=1}{\times} \mathbb{C} P^{n_{1}-1}
$$

is greater than or equal to the greatest common divisor of $n_{1} \cdots n_{k}$.

In the following, we outline the history of Theorems 1 and 2, the method of the proof, and possible generalizations. Note first that as yet, the fixed point problem is the only dynamical problem for elements of $\mathscr{D}$ for which there exist satisfactory conjectures and results (even though the problem of periodic solutions has also been addressed by some authors, see [Z] and [CZ3]). This is not surprising if one compares the principal strategies of a proof of the Lefschetz fixed point theorem and the Arnold conjecture. While the first follows from a finite dimensional geometric argument, the second involves a variational problem on the "infinite dimensional" loop space $C^{\infty}\left(S^{1}, P\right)$, which we will describe next.

Assume that $\psi=\psi_{H, 1}$, where $H$ as in (1) is 1-periodic. Consider the 2-disc $D^{2}$ and identify $\partial D^{2}=S^{\prime}=\mathbb{R} / \mathbb{Z}$. Let $\tilde{z}: D^{2} \rightarrow P$ be a smooth function with boundary values $z$. Then since $d \omega=0$, the integral

$$
\alpha_{H}(\tilde{z})=\int_{D^{2}} \tilde{z}^{*} \omega+\int_{S^{1}} H_{t}(z(t)) d t
$$

depends only on $z$ and the homotopy type of $\tilde{z}$ with fixed boundary. In fact, $a_{H}$ is well defined on the loop space if and only if $I_{\omega}=0$. In any case, we can calculate the derivative

$$
D a_{H}(z) \xi=\int_{S}\left\{\omega_{z(t)}(\dot{z}(t), \xi(t))+d H_{t}(z(t)) \xi(t)\right\} d t,
$$

in the direction of smooth vector fields $\xi$ over $z$. It clearly vanishes for all $\xi$ if and only if $z$ satisfies (1), i.e. if $z(0)$ is a fixed point of $\psi$.

This variational formulation, directly obtained from the principle of least action, 
was used in 1982 by Conley and Zehnder [CZ1] to prove the Arnold conjecture on the torus $T^{2 n}$ with the standard symplectic structure. Note that $\pi_{2}\left(T^{2 n}\right)=0$ and hence $I_{\omega}=0$. Their proof made use of the Conley index applied to the set of bounded trajectories of the gradient flow of $a_{H}$. Although in spirit, this approach applied to all manifolds at least if $I_{\omega}=0$, considerable analytical problems were involved. For example, the application of the Conley index theory as in [C] made it necessary to reduce the variational problem to finite dimensions. In subsequent years the Conley-Zehnder result was generalized (again through finite dimensional reductions of (3)) by Sikorav [S1] and the present author [F1] to certain other quotients of $\mathbb{R}^{2 n}$. In a $C^{0}$-neighborhood of the identity, it was proved for general $P$ by Weinstein [W]. A slightly different point of view was taken in [Ch]: Since the fixed point set of $\psi$ is the intersection of the graph of $\psi$ with the diagonal in $P \times P$, one can reduce the fixed point theory of $\psi$ to an intersection theory of "Lagrangian" submanifolds $L, L^{\prime}$ of a symplectic manifold. (In the same way, the Lefschetz fixed point theorem reduces to cohomological intersection theory of general manifolds.) This intersection theory also has a variational formulation, where the loop space has to be replaced by the space of paths connecting $L$ and $L^{\prime}$. Important results in Lagrangian intersection theory were obtained by Hofer [Ho1], see also [LS].

In 1985, Gromov [G] introduced into symplectic geometry a new set of methods based on the analysis of holomorphic curves. One of the implications was the existence of at least one fixed point for any exact diffeomorphism provided that $I_{\omega}=0$. Gromov used the framework of Lagrangian intersections, which he reduced one step further to the self-intersections of one Lagrangian submanifold $L$ of a symplectic manifold $Q$. He then studied spaces of "holomorphic discs" in $Q$ with boundaries in $L$. The final argument was indirect, but similar methods were used in [F3-7] to prove the Arnold conjecture in the case $I_{\omega}=0$. (Here, the coefficients were restricted to $R=\mathbb{Z}_{2}$.) Working in the framework of Lagrangian intersections, [F3-7] used as a principal tool the space of holomorphic discs whose boundaries lie to equal parts in the two Lagrangians. On the surface, this represents a minor modification to [G]. However, these "cornered" holomorphic discs also have a variational interpretation as the trajectories of the gradient flow of the "Lagrangian" action function. In fact, the proof of [F3-7] fits squarely into the framework of the original approach of [CZ1], representing a new approach to the Conley index which does not require a finite dimensional reduction.

The aim of the present paper is to extend the methods of [F3-7] to cover the case of diffeomorphisms of $P$ where $I_{\omega} \neq 0$. (Here, the Lagrangian framework does not seem appropriate since it is certainly not possible to remove the toplogical restrictions on $L \subset P$ in [F3-7].) The isolated result so far is Fortune's proof [Fo] that every exact deformation on $\mathbb{C} P^{n-1}$ has at least $\operatorname{cl}\left(\mathbb{C} P^{n-1}\right)=n$ fixed points. (For $\mathbb{C} P^{1}=S^{2}$, this result was proved in [Ni] and [Si].) Fortune's proof uses a modification of the variational problem (3) which in turn relies on the fact that $\mathbb{C} P^{n-1}$ is a symplectic quotient (see $[\mathrm{MW}]$ ) of $\mathbb{C}^{n}$. It has so far withstood all attempts to generalize it even e.g. to other symplectic quotients of $\mathbb{C}^{n}$.

Viewed from the variational problem, Fortune's result comes rather as a surprise. If the image of $I_{\omega}$ is cyclic, then the action is a well defined function on the loop 
space taking values in the circles $S^{1}=\mathbb{R} / a \cdot \mathbb{Z}$ for some real number $a$. In finite dimensional Morse theory, one cannot expect such a function to satisfy the Morse inequalities on the quotient; the "correct" statement in this case is given by the Novikov inequalities ([No], see also [S2]). However, Novikov's theory does not apply here, because not only the function, but also the Morse index of a critical point is "ill defined" along loops in the loop space. Of course, this statement does not make any sense in finite dimensions. It is ultimately related to the fact that the usual definition of the Morse index of $a_{H}$ gives an infinite value and that a finite value can only be obtained by comparing two critical points "along" a connecting path, see [V], [F5], or the next section. The indeterminacy in this case is measured by $I_{c}$. In this sense, Theorem 1 states that ambiguity in $a_{H}$ does not effect the Morse inequalities as long as it is proportional to the ambiguity in the Morse index. For more details, we refer to Sect. $1 \mathrm{~b}$.

The proof of Theorem 1 uses the abstract Conley index of [F3-7]. Here, the link between the Morse theoretic approach of [CZ1] and the geometric approach of $[\mathrm{G}]$ is provided by a "geometric Morse theory" which is implicitly contained in [S1] but received little attention until it was rediscovered in [Wi] (see also [F] for a brief review). In Sect. 1b, we give a self contained exposition of the abstract Conley index for (3), formulated as much as possible along the lines of standard Conley index theory (see [C] and [Z2]). The proof of Theorem 2 is given in Sect. 1c. Roughly speaking, we use an analogue of the "refinement" of the Conley index of [F2], which appears here as an operation of tensors in $H^{*}(P)$ on the (abstract) Conley Index. For $I_{\omega}=0$, this operation is defined by the topology of $P$ above. It was shown in [F7] that it yields information about the homology of the underlying invariant set and hence about fixed points. (For a different approach to this problem, see [Ho2]). In general, however, the "refinement" also reflects topological properties of the space of holomorphic spheres in $P$. In fact, it is an interesting example of a symplectic invariant arising from Gromov's theory. For this reason, we have to restrict general fixed point estimates as in Theorem 2 to symplectic manifolds possessing (almost) complex structures for which the holomorphic spheres are known.

The idea of the homological Conley index applies in principle to other problems involving the symplectic action. For example, it is possible to obtain an equivariant version by means of the Borel construction. (Here one uses the fact that the classifying space of a compact Lie group can be approximated by finite dimensional manifolds, which in turn can be added to $\Omega$ without changing the analytic properties of the variational problem essentially.) A difficult problem is often its calculation; e.g. in the case of the Weinstein conjecture on a general contact manifold which cannot be "deformed" to a standard case. (See however [FHV], where the technique of holomorphic curves is used to prove the existence of a closed orbit in a special case. Although [FHV] does not use this notion, it suggests that the (equivariant) homological Conley index is nontrivial in the situation considered.) Another possible application of an equivariant index is the problem of periodic orbits, which must essentially satisfy Morse inequalities in a $\mathbb{Z}_{p}$-equivariant theory.

Applications of the homological index to variational problems other than the symplectic action are rare. While we believe that most variational problems arising 
in differential equations can in principle be formulated by studying trajectory spaces, the strong special features encountered in the next section seem to be limited to those functions whose second derivative is of first order (hence indefinite), and whose nonlinearity is of "borderline" type (in the sense of Palais-Smale, see [Ps]). The only other case meeting these requirements (besides the cubic Dirac equation on surfaces, which looks a little bit like a "constructed" example) is the Chern-Simons function on connections on a 3-dimensional closed manifold, see [F8]. Here, the gradient flow is defined by the selfdual Yang Mills equation on the infinite cylinder $\mathbb{R} \times M$. The motivation in this case is reversed: Rather than proving the existence of critical points (which are the flat connections and therefore have an algebraic interpretation as representations of the fundamental group), one wants to gain information about the original manifold. Despite this difference, the two problems have strikingly similar analytic properties, as we tried to demonstrate in the setup of [F8] and the present paper. Moreover, beyond the variational problem discussed here, the relationship between instantons and holomorphic curves (see also [A2-4]) might indicate a tie between low dimensional and symplectic geometry which has yet to be explored.

1b. The Homological Conley Index. The function $a_{H}$ of (1a.3) is well defined on the Sobolev space $\Omega=L_{k}^{p}\left(S^{1}, P\right)$, see e.g. [K1], for $k>1 / p$. $\Omega$ is a smooth Banach manifold whose charts are given in Sect. 2a. In order to do Morse theory on $\Omega$, we define the gradient flow of $a_{H}$ as follows. Let $J$ be a smooth almost complex structure on $P$ so that $g=\omega(J, \cdot)$ is a metric, i.e. a positive symmetric form, on TP. We will call $J$ an almost Kähler structure and denote the space of all such $J$ by $\mathscr{J}$. Then trajectories of the gradient flow of $a_{H}$ are defined as solutions $u: \mathbb{R} \times S^{1} \rightarrow P$ of the partial differential equation

$$
\left(\bar{\partial}_{J, H}(u)\right)(\tau, t):=\frac{\partial u(\tau, t)}{\partial \tau}+J(u(\tau, t)) \frac{\partial u(\tau, t)}{\partial t}+\nabla_{J} H_{t}(u(\tau, t))=0 .
$$

Here, $\nabla_{J}$ is the gradient with respect to the metric $g$. We sometimes use the short notation

$$
\bar{\partial}_{J, H} u=u^{\prime}+J \dot{u}+\nabla H(u),
$$

where $u^{\prime}$ and $\dot{u}$ denote the $\tau$ - and $t$-derivatives, respectively. The last two terms can be considered as a "vector field" $\mathscr{G}(z)=J \dot{z}+\nabla H(z)$ on $\Omega$ evaluated at $u(\tau)$. It is the $L^{2}$-gradient of $\mathscr{A}_{H}$ in the sense that for $\xi \in T_{z} \Omega$,

$$
D a_{H}(z) \xi=\langle g(z), \xi\rangle=\int_{0} g\left(J \dot{z}(t)+\nabla H_{t}(t, z(t)) \xi(t)\right) d t
$$

by (1a.5). In this way, (1) is the gradient flow equation $u^{\prime}(\tau)=g(u(\tau))$. On the other hand, note that the first two terms in (2) define the Cauchy-Riemann operator $\bar{\partial}_{J} u=u^{\prime}+J \dot{u}$ for maps from the complex manifold $\mathbb{R} \times S^{1}=\mathbb{C} / i \cdot \mathbb{Z}$ into the almost complex manifold $(P, J)$. Hence up to a term of "lower order," the trajectory equation is just the Cauchy Riemann equation, whose solutions are holomorphic maps. Such maps (on compact Riemann surfaces) were first applied to symplectic geometry in $[G]$.

We define the set of paths in $\Omega$ by $\mathscr{P}_{\text {loc }}=L_{1, \mathrm{loc}}^{3}\left(\mathbb{R} \times S^{1}, P\right)$ which embeds in $C^{0}\left(\mathbb{R} \times S^{1}, P\right)$. Since the initial value problem is ill posed for the differential equation 
(2) we define the flow by its "graph,"

$$
\mathscr{F}=\mathscr{F}_{J, H}=\left\{u \in \mathscr{P}_{\text {loc }}(P, M) \mid \bar{\partial}_{J, H}(u)=0\right\},
$$

rather than by a family of maps on a subset of $\Omega$. Note that $a_{H}$ decreases along trajectories in $\mathscr{F}_{J, H}$. (This statement makes sense even though $a_{H}$ is only locally well defined.) In fact, we have for $u \in \mathscr{F}_{J, H}$,

$$
\frac{\partial}{\partial \tau} a_{I I}(u(\tau))=-\left\|g_{J, H}(u(\tau))\right\|_{2}^{2}=-\left\|u^{\prime}(\tau)\right\|_{2}^{2}
$$

Hence the trajectories $u \in \mathscr{F}_{J, H}$ for which

$$
l^{2}(u)=\int_{\mathbb{R} \times S^{1}}\left\|u^{\prime}(\tau)\right\|_{2}^{2} d \tau=\left\|u^{\prime}\right\|_{2}^{2}
$$

is finite are precisely those along which $\alpha_{H}$ is bounded. Now the central notion of our approach can be formulated as follows:

Definition $1 b .1$. For any $(J, H) \in \mathscr{J} \times \mathscr{H}$ and for any closed set $\mathscr{U} \subset \Omega$, we define the Morse complex in $\mathscr{U}$ as the set of bounded trajectories

$$
\mathscr{M}_{J, H}(\mathscr{U})=\left\{u \in \mathscr{F}_{J, H} \mid u(\tau) \in \mathscr{U} \text { for all } \tau \text { and } \iint_{\mathbb{R} S}\left|\frac{\partial u}{\partial \tau}\right|^{2} d t d \tau<\infty\right\} .
$$

Moreover, let $i: \mathscr{F}_{J \pi} \rightarrow \Omega$ be defined by $i u=u(0)$. Then we define the maximal invariant set in $\mathscr{U}$ as

$$
\mathscr{S}_{J, H}(\mathscr{U})=i \mathscr{M}_{J, H}(\mathscr{U}) \subset \Omega \text {. }
$$

We call $\mathscr{S}_{J, H}(\mathscr{U})$ isolated if its closure is contained in the interior of $\mathscr{U}$. In this case, $\mathscr{U}$ is called an isolating neighborhood.

In most applications, $U$ will simply be the space contained in a given closed subset of $P$. The reader who does not feel comfortable with the notion of isolated invariant sets is invited to set $U$ equal to the total loop space of $P$ throughout this paper, since this will suffice for the proof of the theorems of Sect. 1a.

For continuous (local semi-) flows, this notion was developed by Conley [C]. (The parts of Conley's theory to be discussed here are also contained in [CZ2]. However, we do not assume that the reader is familiar with Conley's theory.) The general idea is that certain properties of the flow near an isolated invariant set do not change under continuous deformations of the flow as long as the invariant set remains isolated. These properties can be described by means of homotopy theory. However, Conley's original approach as in [C] and [CZ2] was restricted to the case of compact invariant sets in locally compact spaces. (We will simply refer to this as the finite dimensional case.) In a general infinite dimensional situation, homotopy theory tends to become more difficult in the application and weaker in its assertions. This problem is of course well known also in traditional Morse theory. However, it is overcome more easily since Morse theory only attempts a description of the critical set in terms of the total domain of the function, whereas Conley's theory attempts explicit statements about trajectories joining such critical points. Since the principal tool in either case is the same (the homo- 
topies defined by the flow), the difference might be rather technical. In fact, Conley's theory has been extended, with considerable time delay, to cover many applications in which ordinary Morse theory works (see [RZ]). Indefinite functions like the symplectic action however remained tricky in either approach. In fact, they were hardly considered a useful tool for (nonperturbative) existence results until it was used by Rabinowitz [R] and Weinstein [W] to obtain periodic solutions (for time independent Hamiltonians) on the linear space $\mathbb{R}^{2 n}$. The purpose of this section is to develop a "Conley theory" in this case, with quite different methods, though with remarkably similar results.

To gain a heuristic understanding of our construction, recall the basic idea of $[R]$. Since the infinite dimensional situation did not contain topologically useful information, Rabinowitz restricted the function (1a.3) to the finite dimensional subspaces $E_{v} \subset \Omega\left(\mathbb{R}^{2 n}\right)$ defined by Fourier analysis. For each $N$ he obtained (by a "mountain pass" argument) a critical point $x_{N}$. Since (roughly speaking) the Hamiltonian perturbation compared with the symplectic action becomes less and less significant for high Fourier coefficients, this sequence converges to a critical point of (1a.3), i.e. to a solution of the Hamiltonian equation. The crucial observation is that topologically, increasing $N$ amounts to a suspension, which does not change the situation essentially. In fact, even though the infinite suspension of a topological space may be contractible, the limit of repeated suspensions is topologically meaningful. For example, its homology changes only by a dimensional shift. It was essentially this idea which was applied to the Conley index in [CZ1]. Generalized to other manifolds, it would produce a "stable Conley index," containing the desired homological information. However, it was clear from [F1] that a "global Fourier analysis" on manifolds faces serious obstructions. A definite limit to this approach was set by Gromov's "discovery" of holomorphic spheres: The Fredholm and compactness theory of [G] suggested changes in the variational picture which must in principle elude any finite dimensional approximation (see Sects. 2 and 3). It thereby focused the present author's attention on the new approach of (3), which will be used here to define a "homological" Conley index.

Let us say that an invariant set $\mathscr{S}=\mathscr{S}_{J, H}(\mathscr{U})$ is bounded if the evaluation map $\mathscr{S} \times S^{1} \rightarrow P$ has precompact image. For example, the reader may assume that $\mathscr{U}$ itself is the set of all loops taking values in some compact subset of $P$. In this case, the elliptic nature of the flow equation implies the following result:

Theorem 3. (1) If $P$ is monotone and if $\mathscr{S}_{J, H}(\mathscr{U})$ is bounded and isolated in $\mathscr{U}$, then for all $\left(J^{\prime}, H^{\prime}\right) \in \mathscr{J} \times \mathscr{H}$ close enough to $(J, H)$ in the $C^{\infty}$-topology, $\mathscr{S}_{J^{\prime}, H^{\prime}}(\mathscr{U})$ is isolated in $\mathscr{U}$.

(2) Assume in addition that $I_{\omega}=0$. Then the map i restricted to $\mathscr{M}_{J . H}$ is a homomorphism between the local topology on $\mathscr{P}_{\text {lok }}$ and the induced topology on $\Omega$ for arbitrary Sobolev coefficients. In particular, $\mathscr{F}$ restricts to a continuous flow on $\mathscr{S}_{J, H}$.

Proof. (1) Assume the contrary, i.e. assume that there exists a sequence $\left(J_{\alpha}, H_{\alpha}\right)$ converging to $(J, H)$ in $\mathscr{J} \times \mathscr{H}$ so that $\mathscr{S}_{\alpha}:=\mathscr{S}_{J_{\alpha}, H_{\alpha}}$ is not isolated in $\mathscr{U}$. This means that there exists a sequence $u_{\alpha} \in \mathscr{M}_{\alpha}$ so that $u_{\alpha}(0)=i\left(u_{\alpha}\right)$ is contained in $\mathscr{U}$ but not in its interior. On the other hand, Proposition $3 \mathrm{~b}$ implies that $u_{\alpha} \rightarrow u \in \mathscr{M}_{J, H}$ 
locally in $G^{0}\left(\mathbb{R} \times S^{1} x, P\right)$ for a finite set $x$ in $P$, so that $u_{\alpha}(0) \rightarrow u(0) \in \mathscr{S}_{J, H}$. This contradicts the assumption.

(2) Note that $i$ is injective on all of $\mathscr{F}_{J, H}$ due to Arondzajn's theorem [An] applied to the "square" $(D \bar{\partial})^{+}(u) \bar{\partial}$ of the Cauchy Riemann operator (see also $[\mathrm{McD}])$. The second assertion is again an immediate consequence of Proposition 3b: Since $i$ is obviously continuous, it essentially states that if $u$, $u_{\alpha} \in \mathscr{M}_{J, H}$ and $u_{\alpha}(0) \rightarrow u(0)$ in $\Omega$, then $u_{\alpha} \rightarrow u$ on a neighborhood of $\{0\} \times S^{1}$. Now use the same indirect argument as in the proof of (1).

The appropriate equivalence relation for isolated invariant sets is given by the notion of continuation. Denote by $\bar{J}$ the set of smooth families $\bar{J}=\left\{J_{\lambda}\right\}_{\lambda \in[0,1]}$ of almost Kähler structures. Moreover, denote by $\overline{\mathscr{H}}$ the set of smooth functions on $[0,1] \times S^{1} \times P$.

Definition 1b.2. If $(\bar{J}, \bar{H}) \in \overline{\mathcal{J}} \times \overline{\mathscr{H}}$ and if there exists $\overline{\mathscr{U}} \subset \Omega \times[0,1]$ so that $\mathscr{S}_{\lambda}:=\mathscr{S}_{J_{\lambda} H_{\lambda}}\left(\overline{\mathscr{U}}_{\lambda}\right)$ is bounded and isolated for all $\lambda \in[0,1]$, then $(\bar{S}, \bar{J}, \bar{H})$ defines a bounded continuation between $\mathscr{S}_{0}$ and $\mathscr{S}_{1}$.

To a compact isolated invariant set of a continuous flow $\psi$ on a locally compact space $X$, one assigns its Conley Index, which is defined as follows: For any compact isolating neighborhood of $U$ of $X$, there exist compact sets $A \subset U-S$ satisfying

(I1) If $x \in A$ and $\psi_{[0, t]}(x) \subset U$ for any $t>0$, then $\psi_{[0, t]}(x) \subset A$.

(I2) If $x \in U$ and $\psi_{t}(x) \in U$ for some $t>0$, then $\psi_{[0, t]}(x)$ intersects $A$.

Such a set $A$ is called an exit set for $U$. The pointed homotopy type of the topological quotient $U / A$ with base point $(A)$ is independent of the particular choice of $U$ and $A$ and is called the Conley index $I(S)$ of $S$. Moreover, any continuation as in Definition 1b.3 between two compact isolated invariant sets $S_{0}$ and $S_{1}$ defines a pointed homotopy equivalence between $I\left(S_{0}\right)$ and $I\left(S_{1}\right)$. The aim of this paper is to recover parts of the Conley index theory for $g_{J, H}$-flows on $\Omega$. A direct topological generalization (as given in $[R]$ and $[R Z]$ in certain infinite dimensional situations) does not seem promising in this case. However, for gradient-like flows $\psi$ on finite dimensional manifolds $X$, there exists an interesting geometric construction of the homology $H_{*}(I(S))$ of the Conley index, which can be generalized to our situation. Roughly speaking, if $\psi$ is of "Morse Smale type," then $H_{*}(I(S))$ can be obtained from the free Abelian group $Z_{*}$ over the fixed points of $\psi$ and from a boundary operator $\partial: Z_{*} \rightarrow Z_{*}$, where $\partial$ is defined by trajectories of $\psi$ connecting fixed points, see [F6]. This is a simple generalization of a property of gradient flows on compact manifolds proved essentially by Smale [S1], see also Witten [Wi]. The construction of $\partial$ relies on the following two properties of "generic" (i.e. Morse-Smale) flows:

(S1) The space of trajectories connecting two given fixed points $x$ and $y$ is a smooth manifold $M(x, y)$.

(S2) For any $(u, v) \in M(x, y) \times M(y, z)$, there exists a family $u \#_{\rho} v, \rho \in \mathbb{R}_{+}$, in $M(x, z)$ converging geometrically to $u$ and $v$ as $\rho \rightarrow \infty$. Otherwise, $M(x, z)$ is compact.

We will refer to these properties as "transversality" and "transitivity," respectively. The crucial observation is that even though the space $\Omega$ of our variational problem 
is infinite dimensional, the analysis of the spaces of trajectories connecting two critical points does not differ much from the finite dimensional case. This is summarized in the following result: Let us define $\mathscr{M}\left(x_{+}, x_{-}\right)$as the set of trajectories $u$ in $\mathscr{M}$ such that $u(\tau) \rightarrow x_{ \pm}$in $\Omega$ for $\tau \rightarrow \pm \infty$. Moreover, let us define the set $\mathscr{M}^{k}(x, y)$ of $k$-trajectories from $x$ to $y$ as the set of all $k$-tuples $u_{1} \cdots u_{k}$ so that $u_{i} \in \mathscr{M}\left(x_{i-1}, x_{i}\right)$, where $x_{0}=x, x_{k}=y$.

Proposition 1b. For a dense set in $\mathscr{J} \times \mathscr{H}$ the zero set $\mathscr{Z}$ of $\mathrm{g}$ is nondegenerate and $\mathscr{M}$ decomposes into smooth oriented manifolds $\mathscr{M}(x, y)$ of trajectories connecting $x, y \in \mathscr{Z}$. If $\Gamma \subset \mathbb{Z}$ denotes the set of integrals of the first Chern class of $(T P, J)$ on $\pi_{2}(P)$, then there exists a map $\mu: Z \rightarrow \mathbb{Z} / 2 \Gamma$ so that

$$
\operatorname{dim} \mathscr{M}(x, y)=\mu(x)-\mu(y) \bmod 2 \Gamma .
$$

Moreover, let $\hat{\mathscr{M}}(x, y)=\mathscr{M}(x, y) / \mathbb{R}$ denote the quotient by the translational symmetry. Then if $\mathscr{S}=i \mathscr{M}$ is isolated, there exist local diffeomorphism

$$
\#: \hat{\mathscr{M}}^{k}(x, y) \times \mathbb{R}_{+}^{k-1} \supset \mathcal{O} \rightarrow \hat{\mathscr{M}}(x, y),
$$

which are oriented if the left-hand side is oriented as $\hat{\mathscr{M}}^{k}\left(x_{1}, x_{2}\right) \times \mathbb{R}_{+} \times \hat{\mathscr{M}}\left(x_{1}, x_{2}\right)$ $\times \mathbb{R}_{+} \ldots$, and which satisfy the following properties:

(T1) $\mathcal{O}$ is an open subset such that for each compact $K \subset \hat{\mathscr{M}}^{k}(x, y)$, there exists $\rho(K) \in \mathbb{R}_{+}$with $K \times[\rho(K), \infty) \supset \mathcal{O}$.

(T2) For each $i=1, \ldots, k$, there exists a lifting \# ${ }_{i}$ of \# to $\mathscr{M}(x, y)$ such that the family of maps

$$
z_{\rho}: \hat{\mathscr{M}}^{k}(x, y) \times \mathbb{R} \times S^{1} \rightarrow P, \quad z_{\rho}(u, \tau, t)=\#(u, \underline{\rho})(\tau, t)
$$

converges to the function $u \rightarrow u_{i}(\tau, t)$ in the local $C^{\infty}$-topology for $\rho_{i}, \rho_{i+1} \rightarrow \infty$.

(T3) If $\mathscr{M}$ is bounded and isolated, then the 0 -and 1-dimensional part of $\hat{\mathscr{M}}(x, y)$ are compact up to the image of \#.

Proof. The assertions about the local structure of $\mathscr{M}(x, y)$ in itself follow from the Propositions in Sect. 2a-2c together with the assumption that $\mathscr{M}$ is isolated in some closed set $U$. The transitivity map is constructed in Sect. 2d. Properties (1) and (2) follow directly from Proposition 2d.1. Property (3) follows from Proposition 3b.2.

Parameters $J, H$ satisfying the conclusion of Proposition $1 \mathrm{~b}$ will be called regular. We denote the dimension of $\mathscr{M}(x, y)$ at $u$ by $\mu(u)$. This number is defined by the "spectral flow" of the family $D g(u(\tau))$ through the imaginary axis. (In finite dimensions, it would be equal to $\mu(x)-\mu(y)$, where $\mu(x)$ is the dimension of the negative spectral subspace of $D g(x) g$, i.e. the Morse index). The ambiguity in $\mu$ was calculated in [F5], see also [V]. A related problem is that the compactness properties of $(\mathrm{S} 2)$ can only be partially recovered in the infinite dimensional manifold $\Omega$, since there exists an additional source of noncompactness. A sequence in $\mathscr{M}(x, y)$ may, like a sequence of holomorphic curves in $[\mathrm{G}]$, diverge by splitting off a "holomorphic sphere." (In the related case of harmonic maps from a surface to a Riemannian manifold, this phenomenon was discovered in [SU].) The crucial observation is that in the generic situation, such a splitting can only occur on trajectory spaces $\hat{\mathscr{M}}$ of dimension at least 2 . On the other hand, the construction 
of the homological Conley index will depend only on the zero dimensional components of $\hat{\mathscr{M}}$ which we call isolated trajectories.

Theorem 4. Let $(P, \omega)$ be monotone and let $(J, H)$ be as in Proposition $1 b$. Let $\mathscr{S}=$ $i \mathscr{M}$ be bounded and isolated. Then there are only finitely many isolated trajectories $u$ in $\hat{\mathscr{M}}$. Moreover, let $o(u)= \pm 1$ denote the orientation of $u$ and let $\langle\partial x, y\rangle$ denote the sum of o $(u)$ over all isolated trajectories in $\hat{\mathscr{M}}(x, y)$. Let $Z_{*}$ denote the free abelian group over $\mathscr{Z}$, graded by the Morse index $\mu$. Then the homomorphism

$$
\partial: Z_{*} \rightarrow Z_{*}, \quad \partial x=\sum_{y \in Z}\langle\partial x, y\rangle y,
$$

satisfies $\partial \partial=0$. If we define the $\mathbb{Z} / 2 \Gamma$-graded homology

$$
I_{*}(\mathscr{S}, J, H):=\operatorname{ker} \partial / \operatorname{im} \partial,
$$

then for every bounded continuation $(\overline{\mathscr{S}}, \bar{J}, \bar{H})$ which is regular at the ends there exists an isomorphism

$$
h: I_{*}\left(\mathscr{S}_{0}, J_{0}, H_{0}\right) \rightarrow I_{*}\left(\mathscr{S}_{1}, J_{1}, H\right) .
$$

It is functional with respect to compositions of continuations and invariant under continuous deformations of $(\overline{\mathscr{S}}, \bar{J}, \bar{H})$ within the set of continuations.

Proof. It follows from Proposition $1 \mathrm{~b}$ that the zero dimensional part of $\hat{\mathscr{M}}$ is compact and hence finite. This proves the first assertion. To prove the second assertion, note that the matrix elements

$$
\langle\partial \partial x, z\rangle=\sum_{y \in Z}\langle\partial x, y\rangle\langle\partial y, z\rangle
$$

correspond to the sum of the product signs $o^{2}(u, v)=o(u) o(v)$ over the zero dimensional part of $\hat{\mathscr{M}}^{2}(x, z)$. Now note that by property (T3) of the gluing map, the ends of the 1-dimensional part of $\hat{\mathscr{M}}(x, z)$ are in oriented $1-1$ correspondence with $\left(\mathscr{M}^{2}(x, z), o^{2}\right)$. This proves that (7) vanishes.

The other assertions are proved by a very similar construction applied to families of parameter values $(J, H)$. Rather than stating these properties in the form of Proposition $1 \mathrm{~b}$, we assume that the reader is familiar with chapters 2 and 3 of this paper. To construct the homomorphism $h$, consider the continuation $(\overline{\mathscr{S}}, \bar{H}, \bar{J})$ as a smooth family $\left(\mathscr{S}_{\lambda}, H_{\lambda}, J_{\lambda}\right)_{\lambda \in \mathbb{R}}$ which is constant in $\lambda$ outside $[0,1]$. For critical points $x$ in $\mathscr{S}_{0}$ and $x$ in $\mathscr{S}_{1}$, the solution set $\overline{\mathscr{M}}_{\varepsilon}\left(x, x^{\prime}\right)$ of the equation

$$
\bar{\partial}_{\varepsilon}(u(\tau, t))=\frac{\partial u(\tau, t)}{\partial \tau}+J_{\varepsilon \tau} \frac{\partial u(\tau, t)}{\partial t}+\nabla H_{\varepsilon \tau}(u(\tau, t))
$$

with asymptotics $x$ and $x^{\prime}$ have the same analytical properties as the set $\mathscr{M}$ in Proposition $1 \mathrm{~b}$, except for translational invariance. In fact, the proofs of smothness for the section $\bar{\partial}_{J, H}$, of the Fredholm property in Sect. $2 b$, and of the transversality property in Sect. 2c do not make use of the translational invariance, as long as $\bar{H}, \bar{J}$ is constant outside a compact interval. Hence we only have to make sure that $\mathscr{M}$ does not touch the boundary of $\overline{\mathscr{U}}$ (see Definition 1b.3). This may only be true in the "adiabatic limit," i.e. we may have to choose $\varepsilon$ small in (8). The proof proceeds indirectly: If we assume that for a family of real numbers $\varepsilon$ accu- 
mulating at zero there exists $u_{\varepsilon}$ satisfying $\bar{\partial}_{\varepsilon}(u)=0$ and that some $u\left(\tau_{\varepsilon}\right)$ is contained in $\overline{\mathscr{U}}$ but not in its interior, then Proposition 3a would produce a trajectory in $\mathscr{M}_{\lambda}$ for some $\lambda$ which is contained in the closure of $\mathscr{U}_{\lambda}$ but not in $\mathscr{U}_{\lambda}$. This would violate the assumption that $\mathscr{M}_{\lambda}$ is isolated in $U_{\lambda}$ for all $\lambda$.

The construction of Sect. $2 \mathrm{~d}$ applied to this case yields orientation preserving maps

$\#: \hat{\mathscr{M}}\left(x, x^{\prime}\right) \times \mathbb{R}_{+} \times \hat{\mathscr{M}}\left(x^{\prime}, y^{\prime}\right) \cup \hat{\mathscr{M}}(x, y) \times \mathbb{R}_{-} \times \overline{\mathscr{M}}\left(y, y^{\prime}\right) \supset \mathcal{O} \rightarrow \overline{\mathscr{M}}\left(x, y^{\prime}\right)$

with $\mathcal{O}$ as in (T1) of Proposition 1b. The global compactness theory of Sect. 3, suitably modified, implies again that the 0 - and 1-dimensional part of $\overline{\mathscr{M}}$ is compact up to the image of \#. In algebraic terms, this means that the dimensional part of $\overline{\mathscr{M}}$ defines a homomorphism

$$
h_{\#}(\overline{\mathscr{S}}, \bar{J}, \bar{H}): C_{*} \rightarrow C_{*}^{\prime}
$$

which commutes with $\partial$ and $\partial^{\prime}$.

Now let $(\tilde{\mathscr{S}}, \tilde{J}, \tilde{H})$ be a continuation from $S_{1}$ to $S_{2}$ so that $\tilde{\mathscr{M}}$ has the same properties as $\overline{\mathscr{M}}$ above. If we combine it with $(\overline{\mathscr{S}}, \bar{J}, \bar{H})$ to a continuation $(\widetilde{\widetilde{\mathscr{S}}}, \widetilde{\widetilde{J}}, \widetilde{\tilde{H}})$ with a sufficiently large constant interval in between; then restricted to the zero order parts, we have a bijection

$$
\#: \hat{\mathscr{M}}\left(x, x^{\prime}\right) \times \tilde{\mathscr{M}}\left(x^{\prime}, x^{\prime \prime}\right) \rightarrow \tilde{\tilde{M}}\left(x, x^{\prime \prime}\right)
$$

through another variant of the gluing procedure of Proposition $2 \mathrm{~d}$. (The "gluing parameter" here is the length of the separating interval and therefore does not appear in (10).) This proves that $h_{\#}$ is functional with respect to "regular" continuations.

The next step is to show that $h_{\#}$ does not depend on the generic perturbations involved in the above construction. In fact, if the continuation in turn is deformed continuously depending on a parameter $v \in[0,1]$, then one can show that $\left(h_{0}\right)_{\#}$ and $\left(h_{1}\right)_{\#}$ are chain homotopic. To see this, choose first $\varepsilon$ small enough so that $\overline{\mathscr{M}}_{v}$ is "isolated" in $\bar{U}$ for all $v \in[0,1]$. This is possible for a compact interval. Then consider

$$
\overline{\bar{M}}\left(x, x^{\prime}\right)=\left\{(v, u) \mid v \in[0,1] \text { and } u \in \overline{\mathscr{M}}_{v}\left(x, x^{\prime}\right)\right\} .
$$

The theory of Sects. $1 \mathrm{~b}-1 \mathrm{c}$ is easily modified to incorporate the additional parameters $v$, so that we can assume that $\overline{\bar{M}}\left(x, x^{\prime}\right)$ is a smooth finite dimensional manifold. The analog of Proposition 1d yields maps of type (G2), which again parametrizes all ends of $\overline{\mathscr{M}}$ in dimensions less than 2. Algebraically, this means that the zero dimensional part defines a homomorphism $\gamma: C_{*} \rightarrow C_{*}^{\prime}$ with

$$
\left(h_{1}\right)_{\#}-\left(h_{0}\right)_{\#}=\partial \gamma-\gamma \partial^{\prime} .
$$

This proves that $h_{0}$ and $h_{1}$ induce the same homomorphism in homology.

Now consider the composition of $\overline{\mathscr{S}}$ with its inverse. It can be deformed (through continuations) to the trivial continuation. Since the latter clearly induces the identity on $I_{*}$, this completes the proof of Theorem 4.

Together with Theorem 3, this justifies the following notation:

Definition 1 b.3. For any bounded isolated invariant set $\mathscr{S}$ of $g_{J, H}$ on $\Omega$, we define 
the homological Conley index $I_{*}(S, J, H)$ by applying (6) to a regular perturbation of $H$.

Of course, this definition does not provide a method to calculate $I_{*}$. However, the invariance property allows us in some situations to deform the problem into one which can be reduced to finite dimensions. For example, we have

Theorem 5. (1) If $P$ is monotone and $\mathscr{S}=\mathscr{S}_{J, H}(\Omega)$ is the maximal bounded invariant set, then $I_{*}(\mathscr{S})$ is isomorphic as an Abelian group to $H_{*}(P)$.

(2) Consider on the symplectic product $\widetilde{P}=P \times P^{\prime}$ a product complex structure $\tilde{J}=J \times J^{\prime}$ and a Hamiltonian of the form $\tilde{H}\left(x, x^{\prime}\right)=H(x)+H^{\prime}\left(x^{\prime}\right)$. Then for bounded invariant sets $\mathscr{S}$ in $\Omega$ and $\mathscr{S}^{\prime}$ in $\Omega P^{\prime}$, we have

$$
I_{*}\left(\mathscr{S} \times \mathscr{S}^{\prime}\right)=I_{*}(\mathscr{S}) \otimes I_{*}\left(\mathscr{S}^{\prime}\right) .
$$

Proof. We know from Theorem 3 of [F6] that there exists $J$ so that if $H \in C^{\infty}(P, \mathbb{R})$ is $C^{2}$-small, then all critical loops are constant and every $u \in \mathscr{M}_{J, H}(x, y)$ with $l(u)$ small enough consists of constant loops, i.e. $u(\tau, t)=\bar{u}(\tau)$. In this case, $\bar{u}$ is a trajectory of the gradient flow of $H$ with respect to $g=\omega(J \cdot$,$) . We conclude that$ all $v \in \mathscr{M}(x, y)$ which are not of this form must be topologically obtained from some path of constant loops by the connected sum with a sphere $w$ with $\omega[w]>0$. We show that for monotone $P$, such trajectories do not contribute to the matrix elements. Theorem 5 then follows from Lemma 4.3 of [M], see also [Wi] and Theorem 1 of [F6].

The basic reason why trajectories of nonconstant loops do not contribute to $\partial$ is that because of the additional $S^{1}$-symmetry of loop rotations, such trajectories always come in at least two dimensional families. However, we have to make sure that these families belong to regular Morse cells $\mathscr{M}(x, y)$ in the sense of Proposition 1 b. In order not to destroy the $S^{1}$-invariance, we do not use the abstract perturbations of Proposition 1b, but a perturbation of $J$ as in [McD]. It follows in the same way as in Lemma 9.9 of [McD] that for "generic" almost Kähler structures $J$, all simple trajectories in $\mathscr{M}(x, y)$, i.e. all trajectories which are not of the form $v^{k}(\tau, t)=v(k \tau, k t)$ for some $k>1$, are regular. Hence due to the rotational symmetry, their index is at least 2 , so that they do not contribute to $\partial$. Now we claim that since $P$ is monotone, we have $\mu\left(v^{k}\right) \geqq \mu(v)$. In fact, as we noted before, $v$ is topologically equivalent to the connected sum $u \neq w$ of a trajectory $u$ on $P$ and a sphere $w$ in $P$ with $\omega[w]>0$, and hence $c_{1}[w]>0$. Then $v^{k}$ is topologically equivalent to $u \neq k \cdot w$. Now let $\mu_{H}(x)$ denote the Morse index of $x$ as a critical point of $H$. Then the above claim follows from the following formula of $[\mathrm{V}]$, see also Theorem 3 of [F5]:

$$
\mu(u \# w)=\mu_{H}(x)-\mu_{H}(y)+2 c_{1}(w) .
$$

In the following, we want to summarize the three points in the above construction where we used the monotonicity assumption, and discuss possible adaptations for general symplectic manifolds. We will assume that $\mathscr{U}$ is the full loop space $\Omega$. First, note that in the general case, the set $\mathscr{P}(x, y)$ of paths in $\Omega$ joining two critical points may have infinitely many components of index 1 but of unbounded length. In principle, this might allow the matrix elements $\langle x, \partial y\rangle$ to become infinite while 
in the monotone case, the bound on the action bounds the "complexity" of the homotopy class of trajectories. For example, let $x$ and $y$ be two solutions of (1a.1) in $S^{2} \times \mu S^{2}$, joined by a trajectory of index 1 . Then the flow equation would have index 1 on all spaces $\mathscr{P}_{n}(x, y)$ of trajectories homotopic to trajectories obtained from $\mu_{0}$ by connected sum with $n$ copies of the antidiagonal. The length of a trajectory in $\mathscr{P}_{n}(x, y)$ would satisfy $l(u)=l\left(u_{0}\right)+n(1-\mu)$. One may be able to prove that only finitely many of the sets $\mathscr{M} \cap \mathscr{P}_{n}(x, y)$ count algebraically. However, if no such argument can be found generally, one will have to consider the canonical covering $\widetilde{\Omega}$ of $\Omega$ with covering group $\Gamma=\operatorname{Im}\left(I_{\omega}\right) \subset \mathbb{R}$ and the chain complex

$$
\tilde{Z}_{*}=\left\{\sum_{i=1}^{\infty} \alpha_{i} \tilde{z}_{i} \mid \text { for each } a_{0} \in \mathbb{R} \text {, only finitely many } \tilde{z}_{i} \in \tilde{\mathscr{Z}} \text { satisfy } a\left(\tilde{z}_{i}\right)<a_{0}\right\} \text {. }
$$

It is a free module over $\mathscr{Z}$ of the "weighted" group ring $\Gamma_{*}$ consisting of linear combinations which are finite above each level in $I_{\omega}$. The coboundary property and the invariance of the index should hold.

However, the proof has to be refined slightly, due to a second (and this time seemingly technical) point in this paper where the monotonicity assumption came in. Recall that in the proof of Proposition 1c.1, we argued that in passing to a "weak limit" in $\mathscr{M}$, the index decreases by multiples of 2 since all holomorphic spheres (which necessarily have positive $I_{\omega}$ ) have positive $I_{c}$. Without the monotonicity property, there may actually exist holomorphic spheres of nonpositive Chern number. A family of trajectories splitting off such a sphere would converge weakly to a trajectory of the same or even larger index. As long as the set of holomorphic spheres is regular, i.e. as long as the dimension of the space of holomorphic spheres does not exceed the dimension predicted by the index formula (see Proposition 1c.1 below), such splittings can be excluded by a transversality argument. This is for example the case for (products of) complex projective spaces with the standard complex (but arbitrary symplectic) structure, see also the next section. In general, however, transversality is not necessarily a generic property for such holomorphic spheres which factor through branched coverings of $S^{2}$, see [McD1]. To define the matrix elements in this case requires therefore a suitable modification of the Cauchy Riemann equation in a weak neighborhood of the "weak closure" of $\mathscr{M}(x, y)$. We hope to give a general method for such modifications in the near future.

Monotonicity entered a third time in the calculation of $I_{*}$ in Theorem 5. We do not know if the answer is different in general. However, one should point out that the calculation of $I_{*}$ depends only on the (almost) complex structure and not on the symplectic form.

1c. Cup Products. The cohomology $I^{*}(\mathscr{S})$ of the abstract Conley index is defined as the homology of the dual complex $\left(Z^{*}, \delta\right)$ of $\left(Z_{*}, \partial\right)$. With respect to the canonical basis $\mathscr{Z}$ of $\mathscr{Z}_{*}$, we can identify $Z^{*}$ with $Z_{*}$ and have

$$
\delta: Z^{*} \rightarrow Z^{*} ; \quad \delta(y)=\Sigma x\langle x, \delta y\rangle
$$

with $\langle x, \delta y\rangle=\langle\partial x, y\rangle$. Of course, if the homologies of two complexes coincide, then so do their cohomologies. The advantage of the cohomological notation is that we can define a "cup product" invariant. For the standard Conley index in 
the locally compact case, this is defined as follows. If $(U, A)$ is an index pair for a compact isolated invariant set $S$ of a flow $\chi$ on $X$, then we have $H^{*}(I(S))=H^{*}(U, A)$. Hence we can define

$$
\begin{gathered}
\cup: H^{*}(X) \otimes H^{*}(I(S)) \rightarrow H^{*}(I(S)) \\
\alpha \otimes \beta|\rightarrow \alpha|_{U} \cup \beta .
\end{gathered}
$$

The proof of the invariance of the Conley index is easily adapted to show that $U$ is invariant under continuation, see [F2]. An important property of $\cup$ is that if $\alpha \in H^{*}(X)$ acts nontrivially on $H^{*}(I(S))$, then it does not vanish under the restriction to $S$. This is an immediate consequence of the continuity property of Alexander Spanier cohomology (see [Sp]) and the fact that we can choose the isolating neighborhood $U$ of $S$ arbitrarily small. It can be used to estimate the cuplength of $\mathscr{S}$ and hence the number of fixed points of the restricted flow via Ljusternik Snirelmann theory.

A definition of a similar operation in the loop space could be attempted as follows. Assume that $\alpha \in H^{p}(\Omega)$ is represented by a smooth orientable submanifold of $\Omega$ of codimension $p$. Let $\mathscr{M}$ be regular as in Proposition $1 \mathrm{~b}$ and assume in addition that $\alpha$ meets each cell of $\mathscr{M}$ transversally. Then define

$$
\begin{gathered}
\alpha \cup: Z^{*}(J, H) \rightarrow Z^{*}(J, H) \\
\alpha \cup y=\sum_{x \in \mathscr{M}} x\langle x, \alpha \cup y\rangle,
\end{gathered}
$$

where $\langle x, \alpha \cup y\rangle$ is the intersection number of $\alpha$ with $\mathscr{M}(x, y)$. (Again, we count only the zero dimensional part of $\alpha \cap \mathscr{M}$, so that $\langle x, \alpha \cup y\rangle=0$ unless $\mu(x)-\mu(y)=$ $p \bmod 2 \Gamma$.) In fact, if we assume that $\mathscr{S}$ is actually compact, i.e. if $\mathscr{M}$ has no end splitting off a holomorphic sphere, then we can easily show as in Theorem 4 that all such intersections are finite. We conjecture that $\alpha \cup$ commutes with $\delta(J, H)$, thus defining a homomorphism $\cup: H^{*}(\Omega) \rightarrow \operatorname{End}\left(I^{*}(\mathscr{S} ; J, H)\right)$ which is invariant under compact continuation. Moreover, we conjecture that $\cup$ factors through the restriction homomorphism to $H^{*}(\mathscr{S})$. In fact, this has been shown in [F7] in a slightly different case. If $\mathscr{S}$ is merely bounded, then the compactness properties necessary for the proof of finiteness and invariance of the intersection numbers $\langle x, \alpha \cup y\rangle$ breaks down, since cells of dimension higher than two may have ends splitting off holomorphic spheres. However, it turns out that a very similar program can be carried out if we restrict ourselves to the subring of $H^{*}(\Omega)$ which is pulled back from $H^{*}(P)$ by the zero time map

$$
\text { «: } \Omega \rightarrow P, \quad z(z)=z(0) .
$$

Let us represent cohomology classes $\alpha \in H^{p}(P)$ by the dual singular cycles $\alpha: \cup \Delta^{|\alpha|} \rightarrow$ $P$. Here $|\alpha|=\operatorname{dim}(P)-p$. Define

$$
\alpha \cap \mathscr{M}=\left\{(\lambda, u) \in \cup \Delta^{|\alpha|} \times \mathscr{M} \mid \alpha(\lambda)=u_{P}(0,0) .\right.
$$

Then $\operatorname{dim}(\alpha \cap \mathscr{M})=p-\operatorname{dim} \mathscr{M}$ if $\mathscr{M}$ is a regular cell and $\alpha$ is transverse to the zero map

$$
\varkappa: \mathscr{M} \rightarrow P, \quad z(u)=u(0) \text {. }
$$


Hence if $\operatorname{dim} \mathscr{M}=p$, the matrix elements can be defined as the oriented cardinality of $\alpha \cap \mathscr{M}$, compactness provided. It turns out that the noncompactness can be controlled in the following "generic" situation.

Proposition 1c.1. Assume that the space $\mathscr{S} h_{J}$ of holomorphic spheres decomposes into smooth manifolds of dimension

$$
\operatorname{dim}_{u} \mathscr{S} h_{J}=2 c_{1}(u)+\operatorname{dim} P
$$

such that the map z of (1) and the evaluation map on $\mathscr{S} h_{J}$ meet transversally. Moreover, let $\alpha \in H^{p}(P)$ be represented by a smooth singular cycle which is transverse to the map $s: \mathscr{M} \rightarrow P \times E$ and the evaluation map $\mathscr{S} h_{J} \times S^{2} \rightarrow P$. Then if $\mathscr{S}=i \mathscr{M}$ is bounded and isolated, the zero and one dimensional part of $\alpha \cap \mathscr{M}$ is compact up to the image of the map \# of Proposition $1 b$.

Proof. For any sequence $\left(u_{\alpha}, \lambda_{\alpha}\right) \in \alpha \cap \mathscr{M}(a, b)$ we can (by compactness of $\Delta^{|\alpha|}$ ) choose a subsequence such that $\lambda_{\alpha} \rightarrow \lambda$ in $\Delta^{|\alpha|}$ and $u \rightarrow(u, v) \in \hat{\mathscr{M}} \mathscr{C}(a, b)$. By the properties of weak convergence (see Definition 3a.3), we must have $u \in \hat{M} \cap \alpha$ for some $u \in u$, or some $v \in \underline{v}$ must intersect $\alpha$. However, since all these intersections were assumed to be transverse, we can determine their dimensions and find that they are nonnegative only if the index of $\left(u_{\alpha}, \lambda_{\alpha}\right)$ in $\alpha \cap \mathscr{M}$ is at least 2.

Note that this proof applies only the cohomology classes in $P$ and not to general cohomology classes in $\Omega$. The hypothesis of Proposition 1c.1 is satisfied trivially if $I_{\omega}=0$. As long as we can avoid holomorphic maps $u: S^{2} \rightarrow P$ which factor through a branched covering of $S^{2}$, it is actually a generic property of $J$, see [McD1]. However, to obtain the compactness property of Proposition 1c.1, we need control over the full space $\mathscr{S} h_{J}$. According to the discussion at the end of the preceding section, this is an entirely technical point, so that the following algebraic invariants should be well defined in all situations where we can define $I_{*}$, and should be independent of $J$.

Theorem 6. Let $\mathscr{M}_{J, H}$ and $\mathscr{S} h_{J}$ be regular and transverse in the sense of Proposition 1c.1. Assume in addition that $\mathscr{S}$ is bounded and isolated and let $\langle x, \alpha \cup y\rangle$ denote the (oriented) intersection number of $\alpha$ and $\mathscr{M}(x, y)$. Then the homomorphism $\alpha \cup$ on $Z^{*}$ defined by these matrix elements commutes with $\delta$. The map

$$
\cup: H^{*}(P) \rightarrow \operatorname{End}\left(I^{*}(S)\right)
$$

obtained by perturbation as in Definition $1 b .3$ is well defined. Moreover, if $h$ is the dual of the isomorphism of Theorem 4 corresponding to a bounded continuation between bounded invariant sets $\mathscr{S}_{1}$ and $\mathscr{S}_{0}$ keeping $J$ fixed, and if $\cup_{0}$ and $\cup_{1}$ denote the corresponding operations, then for all $\alpha \in H^{*}(P)$ and $y \in I^{*}(\mathscr{S})$, we have

$$
\alpha \cup_{1} h(y)=h\left(\alpha_{0} \cup y\right) \text {. }
$$

Proof. Finiteness of $\langle x, \alpha \cup y\rangle$ follows directly from Proposition 1c.1. To prove that $\alpha \cup$ commutes with $\delta$, consider any $|\alpha|+1$-cell $N$ in $\mathscr{M}^{k}(x, z)$ and the 1 -dimensional manifold $\alpha \cap N$. By the above, its ends are contained in the image of the gluing map \# of Proposition 1b. In fact, due to the $C^{1}$-approximation property of \#, they are in $1-1$ correspondence to pairs $(u, v) \in(\alpha \cap \mathscr{M} \times \hat{\mathscr{M}})$, where the first 
component has necessarily index $|\alpha|$ and hence the second component is an isolated trajectory. It follows that the oriented sum over such pairs vanishes. On the other hand this sum is by definition of $\delta$ equal to $\langle x,(\alpha \cup \delta-\delta \alpha \cup) y\rangle$.

Now assume that $\alpha=\partial \beta$ for some $\operatorname{singular}(p+1)$-chain $\beta$. Then we claim that $\alpha \cup=\delta \beta \cup+\beta \cup \delta$. This is done in the same way as above by considering the one-dimensional intersection set $\beta \cap N$ for any $|\alpha|$-cell $N$.

If $I_{\omega}=0$, so that in particular $\mathscr{S}$ is compact, $\cup$ has properties very similar to the $\cup$-operation in finite dimensional Conley index theory. In particular, one can show using the methods of [F7] that with $z: S \rightarrow P$ as in (3), we have $z^{*} \alpha \neq 0$ whenever $\alpha \cup$ is nontrivial.

Theorem 7. If $P$ is compact with $I_{\omega}=0$ and if $\mathscr{S}=\mathscr{S}_{J, H}(\Omega)$, then the operation $[P] \cup$ of the top class $[P] \in H^{2 n}(P)$ on $I^{*}(\mathscr{S}) \simeq H^{*}(P)$ corresponds to the cup product in $H^{*} P$. Moreover, we have $*^{*}[P]=0$ in $H^{2 n}(\mathscr{S})$, and in general an injective homomorphism

$$
\varkappa^{*}: H^{*}(P) \rightarrow H^{*}(\mathscr{S}) \text {. }
$$

Proof. Note that the last assertion follows from $z^{*}[P] \neq 0$ by Poincare duality. Now the proof is the same as the proof of Propositions 2.2 and 2.5 of [CE] except that we can use integer coefficients by virtue of the orientations given in Proposition $1 \mathrm{~b}$.

Injectivity of (4) was simultaneously proved by Hofer [Ho2] by a far simpler method which does not involve the homological Conley index. The idea in [Ho2] is to consider the set $\mathscr{M}_{\rho}$ of maps $u$ from the closed manifold

$$
S_{\rho}=D^{2} \bigcup_{S^{1}}\left([-\rho, \rho] \times S^{1}\right) \bigcup_{S^{2}} D^{2} \approx S^{2}
$$

into $P$ satisfying $\bar{\partial}(u)(\tau, t)=\nabla H(u(\tau, t))$ for $|\tau| \leqq \rho$ and $\bar{\partial} u=0$ on the caps. Denote by $\mathscr{S}_{\rho} \subset \Omega$ the restrictions of all $u \in \mathscr{M}_{\rho}$ to the equator $\{0\} \times S^{1}$. Since the equation defining $\mathscr{M}_{\rho}$ is homotopic to the standard Cauchy Riemann equation for contractible maps $S^{2} \rightarrow P$, whose solutions are just the constant maps, one can show tht $z^{*}: H^{*}(P) \rightarrow H^{*}\left(\mathscr{S}_{\rho}\right)$ is injective. On the other hand, $\mathscr{S}_{\rho}$ is contained in any neighborhood $\mathscr{U}$ of $\mathscr{S}$ for $\rho$ large enough. Injectivity of (4) therefore follows from the continuity property of Alexander Spanier cohomology.

One can use this result to obtain estimates on the number of fixed points in $\mathscr{S}$ via Ljusternik-Snirelman theory. However, there also exists a direct geometric way to obtain such estimates. If $I_{\omega}=0$, then it follows immediately from the construction that if $\mathscr{S}_{J, H}$ contains only $k$ critical points for some $J$ and $H$, then for any classes $\alpha_{1} \cdots \alpha_{k} \in H^{*}(P)$ of positive dimension, the operation $\alpha_{1} \cup \cdots \alpha_{k} \cup$ vanishes on $I^{*}(\mathscr{S})$. On the other hand, there is reason to believe that if $I_{\omega}=0$, the operation $\cup$ is "associative" with respect to the cup product on $H^{*}(P)$. This would immediately imply the cuplength estimates in Theorem 1 . However, in view of the results of [F7] and [Ho2], we will not pursue this question here.

On the other hand, if $I_{\omega} \neq 0$, then this geometric method is the only way of estimating the number of degenerate fixed points. We will restrict ourselves here to the following illustrative examples: Let $P=\mathbb{C} P^{n-1}$. It is a matter of fact that 
for the standard complex structure $J$ on $P, \mathrm{Ch}_{J}$ satisfies the hypothesis of Proposition 1c.1. By Theorem 5, we have an isomorphism $I^{*} \simeq H^{*}\left(\mathbb{C} P^{n-1}\right)$. Since the generator $u$ of $\pi_{2}\left(\mathbb{C} P^{n-1}\right)$ has first Chern number $n$, the index is $\mathbb{Z}_{2 n}$-graded with one generator in each even dimension.

Theorem 8. The cup operation of the generator $\alpha$ of $H^{2}\left(\mathbb{C} P^{n-1}\right)$ is an isomorphism $I^{k} \rightarrow I^{k+2}$ for each $k \in \mathbb{Z}_{2 n}$. Moreover, the cup operation is multiplicative with respect to (2) of Theorem 5.

Proof. We use a canonical Morse function for which the cells $\mathscr{M}(x, y)$ can be determined explicitly. For $x \in \mathbb{C}^{n}$, the quadratic function

$$
H(x)=\langle A x, x\rangle=\sum_{i=1}^{n} i\left|x_{i}\right|^{2}
$$

defines a function on $\mathbb{C} P^{n-1}=S^{2 n-1} / S^{1}$ with nondegenerate critical points $Z_{i}=$ $\left[e_{i}\right]$. The gradient flow of $a_{H}$ with respect to the standard complex structure is the projection of the linear flow on the loop space of $\mathbb{C}^{n}=0$ to $\mathbb{C} P^{n-1}$. For $1 \leqq i<n$, the two dimensional component of $\mathscr{M}\left(z_{i}, z_{i+1}\right)$ consists of trajectories of the gradient flow of $H$ and interests the generator $u$ of $H^{2}(P, \mathbb{Z})$ exactly once. The two dimensional component of $\mathscr{M}\left(z_{n}, z_{1}\right)$ is one orbit under the combined translational and rotational group, and also intersect $u$ once.

The phenomenon is most easily understood for $n=2$ and for $H=\varepsilon\langle A x, x\rangle$ with $\varepsilon$ small. Then $\mathscr{M}\left(z_{1}, z_{2}\right)$ is the two-dimensional cell of trajectories on $S^{2}$ connecting the maximum with the minimum of $H$, and $\mathscr{M}\left(z_{2}, z_{1}\right)$ "bifurcates" from the two dimensional set of holomorphic maps from $S^{2}$ to $S^{2}$ fixing the poles. Both sets have a unique intersection with the generator of $H^{2}\left(S^{2}, \mathbb{Z}\right)$, which is represented by any point in $S^{2}$.

Now an obvious indirect argument proves Theorem 2. (Note that the group $\Gamma=c_{1}\left(\pi_{2}(P)\right) \subset \mathbb{Z}$ of covering transformations is generated by the greatest common divisor of the integers $n_{1} \cdots n_{k}$.) For $\mathbb{C} P^{n-1}$, this result was first obtained by Fortune [Fo]. Although Fortune uses very different methods (first converting the problem into an $S^{1}$-invariant variational problem on the loop space on $\mathbb{C}^{n}$ ), the structure of the resulting Morse complex is strikingly similar.

Note that the existence of holomorphic sopheres in $\mathbb{C} P^{n-1}$ was necessary to prove Theorem 8 and hence Theorem 2 . On an "exotic" symplectic structure $\tilde{\omega}$ on $\mathbb{C} P^{n-1}$ with an associated almost Kähler structure $J$ so that $\mathscr{S} h_{J}=\phi$, there would be no topological obstruction against a deformation of $H$ pushing $z_{1}$ and $z_{n}$ together. (Though it is not considered very likely that such $\tilde{\omega}$ exist, one cannot exclude it at this point.) The operation of $H^{2}\left(\mathbb{C} P^{n-1}\right)$ on $I^{*}(S)$ would then not be cyclic but nilpotent. In fact, the cup operation on $I^{*}(S)$ is an example of an algebraic invariant of a symplectic manifold which is derived from $\mathscr{S} h_{J}(P)$ but is independent of $J$ as long as it satisfies the Kähler condition with respect to $\omega$. If this invariant is independent of the topology of $P$, then it must be taken into account when formulating fixed point estimates for exact diffeomorphisms. 


\section{Manifolds of Trajectories}

2a. Analytic Setup. Let exp: $T P \rightarrow P$ denote the exponential map with respect to some metric on $P$. Then for $k>1 / p$ and $x \in \Omega_{k}^{p}$, we have smooth charts

$$
\begin{aligned}
\exp _{x}: T_{x} \Omega_{k}^{p} & =L_{k}^{p}\left(x^{*} T P\right) \supset \mathscr{U} \rightarrow \Omega_{k}^{p} \\
\left(\exp _{x} \xi\right)(t) & =\exp _{x(t)}(\xi(t)),
\end{aligned}
$$

where $\mathscr{U}=\left\{\left(\xi_{P}, \xi_{E}\right) \mid\|\xi\|_{\infty}<r\right\}$ for $r>0$ small enough. The proof of this fact is an obvious extension of the proof of Theorem 9.9 of [K1] to two dimensions and to general Sobolev norms. In the same way, it follows that for any $l \geqq 0$ and $q \geqq 1$ such that $l \leqq k$ and $l-1 / q \leqq k-1 / p$, the family $L_{l}^{q}(x)=L_{l}^{q}\left(x_{P}^{*} T P\right)$ of Banach spaces defines a smooth Banach space bundle $\mathscr{L}_{l}^{q}\left(\Omega_{k}^{p}\right)$ over $\Omega_{k}^{p}$. In fact, the linearization of the chart (1) extends to a smooth set of local trivializations

$$
\begin{gathered}
D \exp : T_{x} \Omega_{k}^{p} \times L_{l}^{q}(x) \rightarrow \mathscr{L}_{l}^{q}\left(\Omega_{k}^{p}\right), \\
D \exp (\zeta, \xi)=D \exp (\xi) \zeta .
\end{gathered}
$$

The aim of this section is to extend the above notions to define Banach manifolds $\mathscr{P}(x, y)$ in the set $\mathscr{P}_{\text {loc }}$ of paths in $\Omega$ defined in (1a.3) and a bundle $\mathscr{L}$ over $\mathscr{P}(x, y)$ in such a way that the flow operator $\bar{\partial}_{J \pi}$ of (1b.1) defines smooth sections of $\mathscr{L}$. The procedure is largely parallel to Sects. 3 and 4 of [F4], though simpler since there are no boundary conditions here, and since we do not have to use "exponentially weighted" norms. Let $l, q$ be Sobolev coefficients such that $L_{k}^{p}\left(S^{2}, \mathbb{R}\right)$ $\subset L_{l}^{q}\left(S^{2}, \mathbb{R}\right)$. Then for $u \in \mathscr{P}_{k}^{p}$, we denote by $\mathscr{L}_{l}^{q}(u)$ the Sobolev space of sections $\xi$ of the pullback bundle $u^{*} T P$ with norm $\|\xi\|_{q, l}=\|\xi\|_{q}+\left\|\nabla^{l} \xi\right\|_{q}$. Here, we use an arbitrary metric and connection. We apply this in particular to the constant path $\hat{x}(\tau, t)=x(t)$.

Definition 2a.1. Let $p>2 / k$. For arbitrary $x, y \in \Omega$, define

$$
\begin{aligned}
\mathscr{P}_{k}^{p}(x, y)= & \left\{u \in \mathscr{P}_{k, \text { loc }}^{p} \mid \text { there exist } \xi \in L_{l}^{p}(\hat{x}) \text { and } \zeta \in L_{l}^{p}(\hat{y})\right. \text { such } \\
& \text { that for } \rho \text { large enough, } u(\tau, t)=\exp _{x} \xi(\tau, t) \text { for } \tau<-\rho \\
& \text { and } \left.u(\tau)=\exp _{y} \zeta(\tau) \text { for } \tau>\rho\right\} .
\end{aligned}
$$

Proposition 2a.1. For any $x, y \in \Omega$ and for $p>2 / k$, the set $\mathscr{P}_{k}^{p}(x, y)$ of Definition $2 a .1$ is a smooth Banach manifold with tangent spaces $T_{u} \mathscr{P}_{k}^{p}(x, y)=\mathscr{L}_{k}^{p}(u)$ and smooth charts

$$
\exp _{u}: \mathscr{L}_{k}^{p}(u) \rightarrow \mathscr{P}_{k}^{p} ; \exp _{u}(\xi)(\tau, t)=\exp _{u(\tau, t)} \xi(\tau, t)
$$

Moreover, for $l \in[0, k]$ and $p \geqq q \geqq 1$ so that $l-2 / q \leqq 1-2 / p$, the Banach spaces $L_{l ;}^{q}(u)$ are fibres of smooth Banach space bundles $\mathscr{L}_{l}^{q}$ over $\mathscr{P}_{\delta}^{p}$ with smooth trivializations

$$
\begin{gathered}
\exp _{u}: T_{u} \mathscr{P} \times L_{l}^{q}(u) \rightarrow \mathscr{L}_{l}^{q}, \\
\exp _{u}(\xi, \zeta)=\mathrm{D} \exp _{u(\tau, t)}(\xi(\tau, t)) \zeta(\tau, t) .
\end{gathered}
$$

The family ex̃ of trivializations allows us to define for any section $\triangleleft$ of $\mathscr{L}_{l}^{q}$ a family of functions $\lrcorner[u]: T_{u} \mathscr{P}_{k}^{p} \rightarrow L_{l}^{q}(u)$ by

$$
\exp _{u}(\xi, \jmath[u](\xi))=\left(\exp _{u} \xi, \triangleleft\left(\exp _{u} \xi\right)\right)
$$


By definition, $\lrcorner$ is $C^{r}$ if and only if the function $\lrcorner[u]$ is $C^{r}$ for all $u$. Moreover, we can define the "covariant derivative..

$$
D\lrcorner(u)=D\lrcorner[u](0) .
$$

Let us now restrict our attention to $\mathscr{P}:=\mathscr{P}_{1}^{3}$ and $\mathscr{L}=\mathscr{L}_{0}^{3}$.

Proposition 2a.2. For $J, H \in \mathscr{J} \times \mathscr{H}$ and for $x, y \in \mathscr{Z}_{J, H}$, formula (1b.1) defines $a$ smooth section of $\mathscr{L}$ over $\mathscr{P}$. Moreover, in the first order expansion

$$
\bar{\partial}_{J, H}[u](\xi)=\bar{\partial}_{J, H}(u)+D \bar{\partial}_{J, H}(u) \xi+N(\xi),
$$

the nonlinear part satisfies the estimates

$$
\begin{gathered}
\|N(\xi)-N(\zeta)\|_{p} \leqq C(u)\left(\|\xi\|_{1, p}+\|\zeta\|_{1, p}\right)\|\xi-\zeta\|_{1, p}, \\
\|N(\xi)\|_{p} \leqq C(u)\|\xi\|_{\infty}\|\xi\|_{1, p} .
\end{gathered}
$$

Here, $C(u)$ is bounded in terms of $\|D u\|_{3}$ and the $C^{2}$-norm of $J$ and $H$.

The proof of smoothness for the transition maps of the charts $\exp _{u}$ and the trivializations $\exp _{u}$ is the same as the proof of the corresponding property in Proposition 3.1 of [F4]. To prove Proposition 2a.2, we first show that the function

$$
\bar{\partial}_{J, H}: T_{u} \mathscr{P} \rightarrow L(u)
$$

is well defined. Since $\bar{\partial}_{J, H} v \in L_{\text {loc }}^{p}(v)$ for all $v \in \mathscr{P}$, it suffices to show that for all $\xi \in L_{1}^{p}(\hat{x})$,

$$
\zeta_{\xi}(\tau)=g_{J, H}[x](\xi(\tau))
$$

defines an element of $L^{p}(\hat{x})$. (Here, we have extended the notation of (2) to sections on $\Omega$.) But this follows from the estimate

$$
\left\|g_{J \pi}[x](\xi(\tau))\right\|_{p} \leqq C\|\xi(\tau)\|_{1, p} .
$$

To prove differentiability, we can treat the sections $\bar{\partial}_{J}$ and $\nabla H$ separately. Even if $\bar{\partial}_{J} u$ and $\nabla H(u)$ do not decay at the ends, their derivatives define continuous operators in $\operatorname{Hom}(T \mathscr{P}, \mathscr{L})$. The first is given by the formula

$$
\left(D \bar{\partial}_{J}\left(u_{P}\right) \xi\right)(\tau, t)=\left(\nabla_{\tau}+J(u(\tau, t)) \nabla_{t}\right) \xi(\tau, t)+\left(\nabla_{\xi(\tau, t)} J\right)(u(\tau, t)) \dot{u}(\tau, t) .
$$

This is a smooth Banach-space bundle homomorphism between $T \mathscr{P}(x, y)$ and $\mathscr{L}$ as in Proposition 3.1 of [F4]. For the perturbation, the linear operator is a matrix operator which even defines a smooth endomorphism of $T \mathscr{P}(x, y)$. The nonlinear estimates can be treated separately for $\bar{\partial}_{J}$ and $\nabla H$, too. We obtain an estimate for the $\bar{\partial}_{J}$-part through Lemma 3.2 of [F4]. The estimate on the Hamiltonian part follows from a $C^{2}$-bound on $H$.

2b. Fredholm Theory. In this section, we examine the relation between the spectrum $\sigma\left(D_{x}\right)$ of the operator $D_{x}=D g_{J, H}(x)$ for $x \in \Omega$ and the linear part $D_{u}=D \bar{\partial}_{J, H}(u)$ of (2a.3) for $u \in \mathscr{P}(x, y)$. First note that $\sigma\left(D_{x}\right)$ is always a discrete subset of $\mathbb{R}$, since $D_{x}$ is self adjoint on $L^{2}\left(x^{*} T \mathscr{P}\right)$ and since for each $a \in \mathbb{R}, D_{x}-a \cdot$ id is a Fredholm operator of degree 0 . 
Proposition 2b. For any $x, y \in \Omega_{k}^{p}$ and for $u \in \mathscr{P}_{k}^{p}(x, y), D_{u}$ extends to a Fredholm operator $D_{u}: L_{l}^{p}(u) \rightarrow L_{l-1}^{p}$ for any $l \leqq k$ if and only if $D_{x}$ and $D_{y}$ have no purely imaginary eigenvalues. To each nondegenerate $\tilde{x} \in \widetilde{\mathscr{Z}}_{J, H}$ in the universal covering of $\Omega$, we can assign an integer $\mu(\tilde{x})$ such that for $u \in \mathscr{P}(\tilde{x}, \tilde{y})$,

$$
\operatorname{Index}(D \bar{\partial}(u))=\mu(\tilde{x})-\mu(\tilde{y}) .
$$

If $c_{1}(\alpha)$ for $\alpha \in \pi_{2}(P)$ denotes the first Chern numbers of $\alpha^{*} T P$, then

$$
\mu(\alpha \# x)=\mu(x)+2 c_{1}(\alpha) \text {. }
$$

Proof. The statements concerning the Fredholm properties of $D_{u}$ follow essentially from Theorem 1.3 of [LM], see also [Ko] and [MP]: By standard Fredholm theory of elliptic operators on compact manifolds (see e.g. [H], Theorem 20.1.2), one can reduce the Fredholm assertion to the case of arbitrarily small paths in $\mathscr{P}(x, x)$ for $x \in \Omega$. Moreover, since the set of Fredholm operators is open in the operator topology, we only have to consider the case of the constant path $\hat{x}$. Since $x$ is nondegenerate, there exists for every $\lambda \in \mathbb{R}$ a resolvent

$$
R_{\lambda}=\left(D_{x}+i \lambda\right)^{-1}: L^{p}(x) \otimes \mathbb{C} \rightarrow L_{1}^{p}(x) \otimes \mathbb{C},
$$

which is bounded independently of $\lambda$. Define

$$
\hat{\xi}(\lambda)=\int e^{i \lambda \tau} \xi(\tau) d \tau
$$

Then

$$
\zeta(\tau)=\frac{1}{2 \pi} \int e^{-i \lambda \tau} R_{\lambda} \hat{\xi}(\lambda) d \lambda
$$

satisfies $D_{\hat{x}} \zeta=\xi$. As in [MP], Theorem 4.1 (see also [Ko] for the case $p=2$ ), we now conclude that $\zeta \in L_{1}^{p}(x)$, so that $D_{\hat{x}}: L_{1}^{p}(\hat{x}) \rightarrow L^{p}(x)$ is bijective with

$$
\|\xi\|_{1, p} \leqq C(x)\left\|D_{\hat{x}} \xi\right\|_{p} .
$$

Let us now write $D_{u}$ in the form

$$
\left(D_{u} \xi\right)(\tau)=\frac{\partial}{\partial \tau} \xi(\tau)+D_{\tau} \xi(\tau)
$$

Then the Fredholm index of $D_{u}$ is given by the "spectral flow" $\mu(u)$ of the operator family $D_{\tau}$ through the imaginary axis. Note that $D_{\tau}$ is self adjoint on $L^{2}$ for all $\tau \in \mathbb{R}$ if $D_{u}=D \bar{\partial}(u)$ is defined as in (2a.2) with respect to the torsion-free metric connection on $P$. If the parametrized spectrum $\sigma(u)=\left\{(\tau, a) \mid a \in \sigma\left(D_{\tau}\right)\right\}$ decomposes into a smooth family of eigenvalues of multiplicity one, then $\mu(u)$ is the number of families crossing zero. A more precise and more general definition of this integer quantity is given as follows: Choose $\tau_{0}<\cdots<\tau_{N}$ and $\lambda_{1} \cdots \lambda_{N}$ in $\mathbb{R}$ so that $\lambda_{i} \notin \sigma\left(D_{\tau}\right)$ for $\tau_{i-1} \leqq \tau \leqq \tau_{i}$ and so that the same is true for $\lambda_{0}:=0$ for $\tau \leqq \tau_{0}$ and for $\lambda_{N+1}:=0$ for $\tau \geqq \tau_{N}$. For $0 \leqq i \leqq N$ we define the integer $n_{i}$ as the dimension of the eigenspace of $D_{\tau_{1}}$ corresponding to $\left[\lambda_{i+1}, \lambda_{i}\right]$ in $\mathbb{C}$ if $\lambda_{i+1} \leqq \lambda_{i}$. Otherwise, we define it to be the negative of the dimension of the eigenspace of $\left[\lambda_{i}, \lambda_{i+1}\right]$. We claim that

$$
\operatorname{Index} D_{u}(\delta)=\sum_{i=0}^{N} n_{i}:=\mu(x, y) \text {. }
$$


This will also prove that the right-hand side is independent of the construction and that it is continuous in $u$. To prove the claim, consider for each $\rho \in \mathbb{R}$ the operator $D(\rho)=(\partial / \partial \tau)+D_{\bar{\beta}_{\rho}(\tau)}$, where $\bar{\beta}_{\rho}(\tau)=\beta(\tau-\rho) \tau+(1-\beta(\tau-\rho)) \rho$. Then we can apply the Fredholm theory described above to $D(\rho)$. In fact, we can extend it to Sobolev norms weighted by a function $\max \left(1, e^{\lambda \tau}\right)$. By Theorem 1.3 of [LM], $D\left(\tau_{i}\right)$ is a Fredholm operator with respect to the weight $\lambda_{i}$ as well as $\lambda_{i+1}$ for $1 \leqq i \leqq N$. By Theorem 1.4 of [LM], the difference of the Fredholm indices in the first and the second case is given by $n_{i}$ (see also Lemma 2e. 2 below). On the other hand, $D(\tau)$ is Fredholm with respect to the weight $\lambda_{i}$ for $\tau_{i-1} \leqq \rho \leqq \tau_{i}$, so that its Fredholm index remains constant through this deformation. Finally, it follows from the above that for large negative $\rho, D(\rho)$ is an isomorphism for the weight 0 . This proves (4).

Since the spectral flow is obviously additive with respect to composition of paths, we obtain the Morse index of (1). Formula (2) for the topological change of the index of $D_{u}$ is given in Theorem 3 of [F5].

2c. Transversality. The aim of this section is to prove the density of regular parameters $(J, H) \in \mathscr{J} \times \mathscr{H}$ of Theorem 5. Since the method depends crucially on the Sard-Smale theorem, we need to restrict the parameter space $\mathscr{H}$ to a Banach manifold. Following [F4], define for a Riemannian manifold $M$ and for every positive sequence $\underline{\varepsilon}=\left(\varepsilon_{i}\right)_{l \in \mathbb{N}}$ the Banach space

$$
C_{\underline{\epsilon}}(M)=\left\{f \in C^{\infty}(M, \mathbb{R}) \mid\|f\|_{\underline{\epsilon}}<\infty\right\},
$$

where

$$
\|f\|_{\underline{\epsilon}}=\sum_{i \in \mathbb{N}} \varepsilon_{i} \cdot \max _{x \in M}\left|\nabla^{i} f(x)\right|
$$

By Lemma 5.1 of $[\mathrm{F} 4]$, we can make $\underline{\varepsilon}$ small enough so that $C_{\epsilon}(M)$ is dense in $L^{p}(M, \mathbb{R})$ for $p<\infty$. For such $\underline{\varepsilon}, \hat{\mathscr{H}}=C_{\epsilon}\left(S^{1} \times P \times E\right)$. Now recall that a subset of a Banach space is said to be of first category if it is a countable intersection of open and dense sets. It is then always dense by Baire's Theorem. We are now ready to state the main results of this section.

Proposition 2c.1. For every $(J, H) \in \mathscr{J} \times \mathscr{H}$, the set $\mathscr{H}_{\text {reg }}(J, H)$ of all $\hat{H} \in H+\hat{\mathscr{H}}$ for which $\mathscr{Z}_{J, \hat{H}}$ is nondegenerate is of first category.

Proof. Consider for some $J \in \mathscr{J}$ the smooth section

$$
\begin{gathered}
\tilde{g}: \hat{\mathscr{H}} \times \Omega \rightarrow \mathscr{L}, \\
\tilde{g}(\hat{H}, x)=g_{J H}(x)+\hat{H},
\end{gathered}
$$

where we identify $\mathscr{L}$ with its pullback under the projection $\hat{\mathscr{H}} \times \Omega \rightarrow \Omega$. Denote by $\tilde{\mathscr{Z}}$ the zero set of $\tilde{g}$. By construction of $\hat{\mathscr{H}}$, the derivative of $\tilde{g}$ with respect to the first factor has a dense image. On the other hand, the derivative of $\tilde{g}$ with respect to the second factor is Fredholm, so that its image is closed with finite codimension. We conclude that $D \tilde{g}$ is surjective for all $(\hat{H}, x) \in \widetilde{\mathscr{Z}}$, and hence that $\tilde{\mathscr{Z}}$ is a $C^{r}$-submanifold 
of $\hat{\mathscr{H}} \times \Omega$, so that the projection $\hat{\mathscr{H}} \times \Omega \rightarrow \hat{\mathscr{H}}$ induces a smooth map $p: \tilde{\mathscr{Z}} \rightarrow \hat{\mathscr{H}}$. The derivatives of $p$ are Fredholm with index zero. In fact, for each $\hat{H} \in \hat{\mathscr{H}}$, we have $p^{-1}[\hat{H}]=\mathscr{Z}_{J, H+\hat{H}}$. Hence $p$ is also sigma proper, so that by the Sard Smale Theorem [S3], see also [Q], the set of regular values of $p$ is of first category in $\hat{\mathscr{H}}$.

Let us now call $\mathscr{M}_{J, H}$ regular if for all $u \in \mathscr{M}_{J, H}(x, y)$, the linearization $D_{u}=$ $D \bar{\partial}_{J \pi}(u)$ is surjective. Of course, this implies by the implicit function theorem that all these sets are smooth manifolds with dimensions given by (2b.1).

Proposition 2c.2. For any $(J, H) \in \mathscr{J} \times \mathscr{H}$, the set of all $\hat{\pi} \in \pi+\hat{\Pi}$ for which $\mathscr{M}_{J, H}$ is regular is of first category.

Proof. We consider the section

$$
\tilde{\partial}: \hat{\mathscr{H}} \times \mathscr{P} \rightarrow \mathscr{L} ; \quad \tilde{\partial}(\hat{H}, u)=\bar{\partial}_{J, H+\hat{H}}(u) .
$$

Again, one easily proves that the zero set $\tilde{\mathscr{M}}$ of $\tilde{\partial}$ is regular. In fact, $D_{2} \bar{\partial}(\hat{H}, u)=$ $D \bar{\partial}_{J, H+H}(u)$ is Fredholm for the values of $\delta$ which we consider. Its finite dimensional cokernel can be removed by means of $D_{1} \bar{\partial}(\hat{H}, u)$. Now Proposition 2c.2 follows as Proposition 2c.1 from the Sard Smale Theorem.

$2 d$. Transitivity. Let $\beta: \mathbb{R} \rightarrow[0,1]$ be a smooth function with $\beta(\tau)=0$ for $\tau \leqq 0$ and $\beta(\tau)=1$ for $\tau \geqq 1$. Consider $\chi=(u, v, \rho) \in \hat{\mathscr{M}}(x, y) \times \hat{\mathscr{M}}(y, z) \times \mathbb{R}_{+}$. If $\rho$ is large enough so that $u(\tau)=\exp _{y} \xi(\tau)$ for $\tau \geqq \rho$ and $v(\tau)=\exp _{y} \zeta(\tau)$ for $\tau \leqq-\rho$, then we define

$$
w_{\chi}=u \hat{\#}_{\rho} v= \begin{cases}u(\tau+\rho) & \text { for } \tau \leqq-1 \\ \exp _{y}(\beta(-\tau) \xi(\tau+\rho)+\beta(\tau) \zeta(\tau-\rho)) & \text { for }|\tau| \leqq 1 \\ v(\tau-\rho) & \text { for } \tau \geqq 1\end{cases}
$$

More generally, recall the set $\hat{\mathscr{M}}_{J \pi}^{k}(x, y)$ of $k$-trajectories joining $x$ and $y$, i.e. the set of $k$-tuples $\underline{u}=\left(u_{1} \cdots u_{k}\right)$ with $u_{i} \in \hat{\mathscr{M}}_{J \pi}\left(x_{i-1}, x_{i}\right)$, where $x=x_{0}$ and $y=x_{k}$. Then we can extend the above construction to a map

$$
\begin{aligned}
& \#: \hat{\mathscr{M}}^{k}(x, y) \times \mathbb{R}_{+}^{k} \supset K \times\left[\rho_{K}, \infty\right)^{k-1} \rightarrow \mathscr{P}(x, y),
\end{aligned}
$$

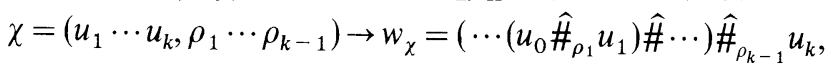

as long as $K$ is compact and $\rho_{K}$ is large enough, see Theorem 3b.1 below. Moreover, it follows from Theorem $3 \mathrm{~b} .1$ that there exists $C_{K} \in \mathbb{R}_{+}$so that

$$
\left\|\bar{\partial}_{J \pi}\left(w_{\chi}\right)\right\|_{p} \leqq \sum e^{-C_{K} \rho_{l}}
$$

Proposition 2d.1. For any compact set $K \subset \hat{\mathscr{M}}^{k}(x, y)$ contained in the regular set, there exist positive real numbers $\rho_{K}, C_{K}$ and a map

$$
\begin{gathered}
K \times\left[\rho_{K}, \infty\right)^{k-1} \rightarrow \hat{\mathscr{M}}, \\
\chi=(\underline{u}, \underline{\rho}) \mid \rightarrow u_{1} \#_{\rho_{1}} u_{1} \# \cdots \#_{\rho_{k-1}} u_{k}=\exp _{w_{\chi}} \xi,
\end{gathered}
$$

with $\|\xi\|_{1, p} \leqq \sum_{i=0}^{k} e^{-C_{K} \rho_{l}}$. Moreover, \# satisfies (1) of Theorem 4. 
The proof of Proposition 2d.1 uses an iterative procedure known as Picard's method, which was first applied to partial differential equations by C. Taubes. It can be summarized as follows:

Lemma 2d.1. Let $f: E \rightarrow F$ be a $C^{1}$-map between Banach space $E$ and $F$. Assume that in the first order expansion

$$
f(\xi)=f(0)+D f(0) \xi+N(\xi)
$$

$D f(0)$ has a finite dimensional kernel and a right inverse $G$ so that for $\xi, \zeta \in E$

$$
\|G N(\xi)-G N(\zeta)\|_{E} \leqq C\left(\|\xi\|_{E}+\|\zeta\|_{E}\right)\|\xi-\zeta\|_{E}
$$

for some constant $C$. Set $\varepsilon=(5 C)^{-1}$. Then if $\|G f(0)\|_{E} \leqq \varepsilon / 2$, there exists a $C^{1}$-function

$$
\phi: K_{\varepsilon}:=\left\{\xi \in \operatorname{ker} D f(0) \mid\|\xi\|_{E}<\varepsilon\right\} \rightarrow G F
$$

with $f(\xi+\phi(\xi))=0$ for all $\xi \in K_{\varepsilon}$. Moreover, we have estimates

$$
\begin{gathered}
\|\phi(0)\|_{E} \leqq 2\|G f(0)\|_{E} \\
\|D \phi(0) \zeta\|_{E} \leqq 8\|G f(0)\|_{E}\|\zeta\|_{E} .
\end{gathered}
$$

The proof of Lemma $2 \mathrm{~d} .1$ is an elementary application of the contraction principle. We apply it here to the function

$$
\begin{aligned}
f_{\chi} & :=\bar{\partial}_{J, H}\left[w_{\chi}\right]: L_{1}^{p}\left(w_{\chi}\right) \rightarrow L^{p}\left(w_{\chi}\right) ; \\
f_{\chi}(\xi) & =\bar{\partial}_{J, H} w_{\chi}+D_{\chi} \xi+N_{\chi}(\xi),
\end{aligned}
$$

see Proposition 2a.2. The absolute and the nonlinear parts satisfy the estimates (2a.4) and (2) with constants that depend only on $K$. Hence Lemma 2 d.1 yields the desired map if we can invert $D_{\chi}$ in a uniform way. Of course, since $\operatorname{Index}\left(D_{\chi}\right)=$ $\mu(x)-\mu(y)>0$, we want to factor out a finite dimensional subspace of $L_{1}^{p}\left(w_{\chi}\right)$. We therefore consider the linear analogue of (1): For $\xi \in L_{1}^{p}(u)$ and $\zeta \in L_{1}^{p}(v)$ we define $\xi \# \zeta \in L_{1}^{p}\left(u \hat{\#}_{\rho} v\right)$ by

$$
(\xi \# \zeta)(\tau)=\left\{\begin{array}{lll}
\beta(\tau-1) \zeta(\tau-\rho) & \text { for } & \tau \geqq 1 \\
\beta(-\tau-1) \xi(\tau+\rho) & \text { for } & \tau \leqq 1
\end{array}\right.
$$

Again, we can extend this to a linear map

$$
\#: L_{k}^{p}\left(u_{1}\right) \times \cdots \times L_{k}^{p}\left(u_{k}\right) \rightarrow L_{k}^{p}\left(u_{1} \hat{\#}_{\rho_{1}} \cdots \hat{\#}_{\rho_{k-1}} u_{k}\right)
$$

for $k=0,1$. Now define $L_{\chi}^{\perp}$ as the $L^{2}$-orthogonal complement of $\operatorname{ker} D_{u_{1}} \# \cdots \# \operatorname{ker} D_{u_{k}}$ in $L_{1}^{p}\left(w_{\chi}\right)$. (This subset is in $L^{2}\left(w_{\chi}\right)$ by Proposition 3 c.) Then we have

Lemma 2d.2. There exist constants $C_{K}$ and $\rho_{K}$ such that if $\chi \in K \times\left[\rho_{K}, \infty\right)^{k-1}$, then there exists a continuous right inverse $G_{\chi}: L^{p}\left(w_{\chi}\right) \rightarrow L_{\chi}^{\perp} \subset L_{1}^{p}\left(w_{\chi}\right)$ of $D_{\chi}$ with

$$
\left\|G_{\chi} \xi\right\|_{1, p} \leqq C_{K}\|\xi\|_{p}
$$


Proof. In a slightly different case and for only two trajectories, this is Lemma 4.3 of [F3]. The proof given here follows the same lines. Since the Fredholm index of $D_{\chi}$ restricted to $L_{\chi}^{\perp}$ is zero, it suffices to show that for all $\xi \in L_{\chi}^{\perp},\|\xi\|_{1, p} \leqq$ $C\left\|D_{\chi} \xi\right\|_{p}$ with $C$ depending only on $K$. This is done indirectly. Assume that there exists a sequence $\chi_{\rho}=\left(\underline{u}_{\rho}, \underline{\rho}_{\rho}\right) \in K \times[\rho, \infty)^{k-1}$ for $\rho \in \mathbb{N}$ and $\xi_{\rho} \in L_{\chi_{\rho}}^{\perp}$ satisfying

$$
\begin{aligned}
\left\|\xi_{\rho}\right\|_{1, p} & =1, \\
\lim _{\rho \rightarrow \infty}\left\|D_{\ell \rho} \xi_{\rho}\right\|_{p} & =0 .
\end{aligned}
$$

We will henceforth abbreviate the double index $\chi_{\rho}$ to $\rho$. We first show that $\xi_{\rho}$ is small near the gluing points. Choose therefore $\tau_{1 \rho} \cdots \tau_{k-1, \rho}$ so that

$$
w_{\rho}\left(\tau_{i \rho}-\rho_{i \rho}\right)=u_{i}(0)
$$

i.e. so that $w_{\rho}\left(\cdot-\tau_{i \rho}\right)=u_{i-1} \hat{\#}_{\rho_{i}} u_{i}$ on $[-\zeta, \zeta] \in \mathbb{R}$. Now define $\xi_{i \rho}(\tau)$ for $|\tau| \leqq 3$ by

$$
D \exp p_{x_{i}}\left(\zeta_{i}(\tau)\right) \xi_{i \rho}
$$

where $\zeta_{i}$ is defined so that $\exp _{x_{i}} \zeta_{i}(\tau)=w_{\rho}(\tau)$ on this interval. Our first aim is to show that for each $i$,

$$
\left\|\xi_{i \rho \mid[-3,3]}\right\|_{1, p} \rightarrow 0 .
$$

Note therefore that $\xi_{i \rho}$ is defined by (6) on increasing intervals $I_{\rho} \uparrow \mathbb{R}$. With $\beta_{(\rho)}(\tau)=\beta(\tau+\sqrt{\rho}) \cdot \beta(-\tau+\sqrt{\rho})$ the sequence $\beta_{(\rho)} \xi_{i \rho}$ is bounded in $L_{1}^{p}\left(\hat{x}_{i}\right)$ by (4). Hence a subsequence converges weakly in this space to a limit $\xi_{i \infty}$. By weak continuity of the nonlinear part $N$ of $\bar{\partial}_{J \pi}$, (see (2a.4) of Proposition 2a.2), we conclude that $\xi_{i \infty}$ satisfies the translationally invariant equation $D_{\hat{x}_{i}} \xi_{i \infty}=$ $(\partial / \partial \tau) \xi_{i \infty}+A_{x_{i}} \xi_{i \infty}=0$. It follows by (2b.3) that $\xi_{i \infty}=0$, so that a compact Sobolev embedding implies that $\xi_{i \rho}$ converges to 0 uniformly on bounded intervals of $\mathbb{R}$. With $\bar{\beta}(\tau)=\beta(\tau+4) \beta(-4+\tau)$, we have $\left\|D_{\hat{x}_{i}}\left(\bar{\beta} \xi_{i \rho}\right)\right\|_{p} \leqq\left\|D_{\hat{x}_{i}} \xi_{i \rho \mid[-4,4]}\right\|_{p}+$ $\left\|\beta^{\prime} \xi_{i \rho}\right\|_{p}$ which converges to zero for large $\rho$ by the above. Hence (7) follows from (2b.3).

Now define $\sigma_{j \rho} \in \mathbb{R}$ so that $w_{\rho}\left(\tau-\sigma_{j \rho}\right)=u_{j \rho}(\tau)$ for $\tau \in I_{j \rho}:=\left[1-\rho_{j-1}, \rho_{j}-1\right]$. Define the cutoff function $\beta_{j \rho}=\beta\left(\tau+\rho_{j-1}\right) \beta\left(-\tau+\rho_{j}\right)$. Then the support of $\beta_{j \rho}^{\prime}$ corresponds to the bounded set where $\xi_{\rho}$ converges to zero by (7). Hence

$$
\left\|D_{u_{j \rho}} \beta_{j \rho} \xi_{j \rho}\right\|_{p} \leqq\left\|D_{u_{j \rho}} \xi_{j \rho}\right\|_{p}+\left\|\beta^{\prime} \xi_{j \rho}\right\|_{p}
$$

converges to zero by (5) and (7). Now there exists a constant $C_{K}$ depending only on $K$ so that if $p_{\rho}$ denotes the $L^{2}$-projection onto the kernel of $D_{u_{\rho}}$, we have

$$
\left\|\beta_{j \rho} \xi_{j \rho}\right\|_{1, p} \leqq C\left(\left\|D u_{j \rho} \beta_{j \rho} \xi_{j \rho}\right\|_{p}+\left|p_{\rho} \beta_{i \rho} \xi_{i \rho}\right|\right)
$$

This converges to zero by the above and since $\xi_{\rho}$ was in the $L^{2}$-complement of ker $D_{u_{1 \rho}} \hat{\# \cdots}$ \#ker $D_{u_{k \rho}}$, see (1). It follows with (7) that $\left\|\xi_{\rho}\right\|_{1, p} \rightarrow 0$ in contradiction to (4). This completes the proof of Lemma $2 \mathrm{~d} .2$.

We can now construct the correction term $\xi$ of Proposition 2d.1. To prove that the map \# is $C^{r}$, note that this is true for the map \# from $K \times\left[\rho_{k}, \infty\right)$ to $\mathscr{P}(x, y)$. 
Hence it suffices to show that $\hat{\#}$ is everywhere transverse to $L_{\chi}^{\perp}$. This is the case since one can show that if $\alpha \in T_{\chi}\left(K \times\left[\rho_{k}, \infty\right)\right) \subset \bigoplus_{i=1}^{k} \operatorname{ker} D_{u_{i}}$, then $\alpha^{\prime}=(D \hat{\#})(\chi) \alpha$ satisfies $\left\|D_{u} \alpha^{\prime}\right\|_{p} \leqq \varepsilon_{\chi}\|\alpha\|_{1, p}$ with $\varepsilon_{\chi} \rightarrow 0$ if all $\rho_{i} \rightarrow \infty$. This also proves the estimate on the

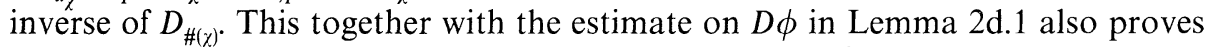
the $C^{1}$-convergence in (2) of Proposition $1 \mathrm{~b}$ in the $M^{k}$-directions. $C^{\infty}$-convergence in general follows from regularity theory on the elliptic equation $\xi=-G\left(\bar{\partial} w_{\chi}+N_{\chi}(\xi)\right)$.

2e. Orientations. To construct the orientations of Proposition $1 \mathrm{~b}$, we find it convenient to rewrite \# in the "unreduced" form. Consider therefore the diffeomorphism $\mathscr{M} \rightarrow \hat{\mathscr{M}} \times \mathbb{R}, u \rightarrow(\hat{u},\langle u\rangle)$ given by $u=\hat{u}(\cdot-\langle u\rangle)$ for some "slice" $\hat{\mathscr{M}} \subset \mathscr{M}$. Then with $\rho=\Sigma\left\langle u_{i}\right\rangle$ and $\rho_{i}=\frac{1}{2}\left(\left\langle u_{i}\right\rangle+\left\langle u_{i+1}\right\rangle\right)$, we can define on some open subset

$$
\#: \mathscr{M}^{k}(x, y) \rightarrow \mathscr{M}(x, y) ; \quad u_{1} \# \cdots \# u_{k}=\#(\underline{u}, \underline{\rho})(\cdot-\rho) .
$$

In Morse theory on a finite dimensional manifold $M$, a frame of the normal bundle of $\mathscr{M}(x, y)$ is naturally given in terms of frames of the negative subspaces $E_{x}^{-}$of the Hessian at the critical points. In fact, they define frames on $T W^{u}(x)$ and on the normal bundle $T^{\perp} W^{s}(x)$, which in turn define a frame on the complement of $T \mathscr{M}(x, y)=T\left(W^{u}(x) \cap W^{s}(y)\right)$ in the trivial bundle $\left.T M\right|_{\mathscr{M}(x, y)}$. Of course, since the group of linear homeomorphisms of an infinite dimensional Hilbert space is contractible by Kuiper's theorem [Ku], we do not have an invariant notion of framing on $E_{x}^{-}$in the infinite dimensional case. In fact, we will see that the structure group of $T \mathscr{M}(x, y)$ cannot in general be (stably) reduced to the trivial group.

Define the manifold $Z_{+}=\mathbb{R}_{+} \times S^{1} \cup D^{2}$. Consider $x \in Z_{J, H}$ and the space

$$
\begin{aligned}
\mathscr{P}(x)= & \left\{u \in L_{2 ; \text { loc }}^{2}\left(Z_{+}, P\right) \mid \text { there exists } \xi \in L_{2}^{2}(\hat{x}),\right. \\
& \text { so that for } \left.\tau \text { large enough, } u(\tau, t)=\exp _{x} \xi(\tau, t)\right\}
\end{aligned}
$$

of one sided paths ending at $x$, see Definition 2a.1. Clearly, we can give $\mathscr{P}(x)$ a Banach manifold structure as in Proposition 2a.1. Moreover, as in Proposition 2a.2, we have a smooth section defined by

$$
\bar{\partial}_{J, H}(u)(\tau)=\bar{\partial}_{J} u(\tau)+\beta(\tau) \nabla H(u(\tau)) .
$$

Here, the Cauchy Riemann operator has been extended to $Z_{+}$. Recall that $\beta(\tau)=0$ for $\tau \leqq 0$ and $\beta(\tau)=1$ for $\tau \geqq 1$. Let us denote by $D(u)$ the "covariant" derivative of $\bar{\partial}_{J, H}$ with respect to local trivializations of $\mathscr{L}$ defined as in Proposition 2a.1. We extend it to a Fredholm operator $L_{1}^{2}(u) \rightarrow L^{2}(u)$. It defines a map $D: \mathscr{P}(x) \rightarrow \mathscr{F}_{\mathbb{R}}$, where $\mathscr{F}_{\mathbb{B}}$ is the set of Fredholm operators on the Hilbert space homeomorphic to $L_{1}^{2}(u)$ and $L^{2}(u)$. ( $D$ is well defined up to homotopy, since as we already remarked above the group of homeomorphisms of a Hilbert space is contractible.) Let $\mathscr{F}_{\mathbb{C}}$ denote the space of Fredholm operators on an infinite dimensional separable complex Hilbert space. 
Lemma 2e.1. There exists a lifting

$$
D \simeq \tilde{D} \circ i: \mathscr{P}(x) \rightarrow \mathscr{F}_{\mathbb{C}} \subset \mathscr{F}_{\mathbb{R}} .
$$

Proof. Consider for any $x \in P$ the space

$$
\Omega^{2} P=\left\{u \in L_{2}^{2}\left(S^{2}, P\right) \mid u(0)=x\right\} .
$$

The linearization of the Cauchy Riemann operator yields an elliptic differential operator

$$
D \bar{\partial}(u): L_{2}^{2}\left(u^{*} T P\right) \rightarrow L_{1}^{2}\left(\bar{H}_{u}\right),
$$

where $\bar{H}_{u}$ is a certain linear bundle over $u$, see [G]. Given a local chart $z=$ $t+i \tau$ of $S^{2}, \bar{H}_{u}$ can be identified with $u^{*} T P$. We then have

$$
D \bar{\partial}(u) \xi=\nabla_{\tau} \xi+J \nabla_{t} \xi+A \xi,
$$

where $A$ is a zero order operator which depends on the connection and on the system of charts with respect to which $D \bar{\partial}(u)$ is defined. If we choose $\nabla$ to be a Hermitian connection, i.e. if we assume that $\nabla J, \nabla \omega$, and $\nabla g$ vanish, then we see that (2) defines a canonical deformation of $D \bar{\partial}(u)$ into a complex linear operator $D_{0}(u)=\nabla_{\tau}+J \nabla_{t}$. Hence if we identify the Banach spaces in (3) as complex Banach spaces, we obtain a map $D_{0}: \Omega^{2} P \rightarrow \mathscr{F}_{\mathbb{C}}$.

Now for any $C^{1}$-map $\bar{x}: D^{2} \rightarrow P$, so that $\left.\bar{x}\right|_{\partial D^{2}}=z$ and $\bar{z}(0)=x$, we obtain a map

$$
\begin{gathered}
\psi: \mathscr{P}(x) \rightarrow \Omega^{2} P, \\
\psi(u)(\theta)=\left\{\begin{array}{lll}
\bar{x}(\theta) & \text { for } & \theta \in D^{2} \\
u(\theta) & \text { for } & \theta \in S^{2}
\end{array}\right.
\end{gathered}
$$

where $S^{2}=S \cup D^{2}$ with $\partial S=\partial D^{2}=S^{1}$ identified. In fact, $\psi$ is a homotopy equivalence, since the $E$-component of $\mathscr{P}(x)$ is contractible. We claim that the diagram

$$
\begin{gathered}
\mathscr{P}(x) \stackrel{D}{\longrightarrow} \mathscr{F}_{\mathbb{R}} \\
\psi \downarrow \\
\Omega^{2} P \underset{D_{0}}{\longrightarrow} \mathscr{F}_{\mathbb{C}}
\end{gathered}
$$

is homotopy commutative up to a homotopy equivalence of $\mathscr{F}$ which changes the Fredholm index by a constant. To see this, note that the disc $\bar{x}$ which we used to construct the homotopy equivalence $\psi$ defines a family $x_{\tau} \in \Omega$ connecting $x$ with $x_{0}$. We therefore obtain a family $\psi_{\tau}: \mathscr{P}(x) \rightarrow \mathscr{P}\left(x_{\tau}\right)$ of homotopy equivalences so that $\psi_{1}=\mathrm{id}$ and $\psi_{0}=\psi$, which in turn defines a family of elliptic operators $D_{(\tau)}(u)=$ $D \bar{\partial}$. (Recall from the proof of Proposition 2a.2 that it is not necessary that $g_{J, H}\left(x_{\tau}\right)$ vanishes.) Next, by switching off the perturbation, we deform the operator field $D(0):=D\left(\psi_{1} u\right)$ into $D(-1)=D_{0}\left(\psi_{1} u\right)$ which is defined in the same way as $D_{0}$ in (4) with $S^{2}$ replaced by $S$. Of course, these operators do no remain Fredholm operators on $T P$, since the asymptotic operators may develop kernels along 
the deformation. However, using the exponential weights of $[L M]$, which we already encountered in the proof of Proposition $2 \mathrm{~b}$, and the following Lemma 2e.2, we obtained successive homotopy equivalences between $D \bar{\partial}$ on $\mathscr{P}(u)$ and $D_{0}$ on $\mathscr{P}_{\varepsilon}\left(x_{0}\right)$ which complete the proof of Lemma 2e.1.

Lemma 2e.2. For $\lambda \in \mathbb{R}-\sigma\left(D_{x}\right)$, denote by $D_{\lambda}: \mathscr{P}(x) \rightarrow \mathscr{F}_{\mathbb{R}}$ the map $D$ with respect to the exponential weight $\lambda$. Then for every $\lambda<\mu \in \mathbb{R}-\sigma\left(D_{x}\right)$ there exists a homotopy equivalence $h: \mathscr{F}_{\mathbb{R}} \rightarrow \mathscr{F}_{\mathbb{R}}$ such that the maps $D_{\lambda}$ and $h \circ D_{\mu}$ are homotopic. Moreover, $h$ increases the Fredholm index by the dimension of the eigenspace $E(\lambda, \mu)$ of $D_{x}$ corresponding to $[\lambda, \mu]$.

Proof. It obviously suffices to prove Lemma $2 \mathrm{e} .2$ for the subspace of such $u \in \mathscr{P}(x)$ which satisfy $u(\tau)=x$ for $\tau \geqq 1$. Moreover, one can reduce it to the following statement: Assume that $E_{u} \subset L^{p}(u)$ defines a finite dimensional subbundle $E$ of $\mathscr{L}^{p}$ so that each $\xi \in E_{u}$ satisfies $\xi(t)=\mathrm{O}$ for $\tau \geqq 1$. Let $\pi$ denote the $L^{2}$-orthogonal projection onto the $L^{2}$-complement of $E$. Then it suffices to show that for all such $E$, we have a continuous splitting of bundles $\operatorname{ker} \pi d_{2}=\operatorname{ker} \pi D_{1} \oplus E(\lambda, \mu)$ over the set of $u \in(\mathscr{P}(x))$ so that $\pi D_{1}(u)$ is surjective. This splitting is easily obtained through the map assigning to each $\xi \in \operatorname{ker} \pi D_{2}$ the projection of $\xi(1)$ onto $E(\lambda, \mu)$. In fact, the projection of $\xi(1)$ onto $E(\lambda, \infty)$ must vanish if $|\xi(\tau, t)| \leqq e^{-\mu \tau}$. Moreover, if $\xi(1)$ $\in E(-\infty, \lambda)$, then $|\xi(\tau, t)| \leqq e^{-\mu \tau}$.

Now recall that $\mathscr{F}_{\mathbb{R}}$ and $\mathscr{F}_{\mathbb{C}}$ are the classifying spaces for the orthogonal and the unitary group, respectively. Hence it follows from Lemma 2e.1 and the family's index theory of $[\mathrm{ASI}]$ that $\mathscr{P}(x)$ is oriented in the sense that for each $u \in \mathscr{P}(x)$, and for each finite dimensional subspace $E \subset L^{p}(u)$ not annihilated by any element of $\operatorname{cok} D \bar{\partial}(u)$, the manifold

$$
\mathscr{M}_{E}(x)=\left\{\xi \in T_{u} \mathscr{P}(x) \mid \bar{\partial}[u](\xi) \in E\right\}
$$

(with $\bar{\partial}[u]$ as in (2a.2)) has a natural orientation given an orientation of $E$. To define the orientations on the Morse complex, we extend the gluing map (1) to incorporate the spaces $\mathscr{M}^{E}$. For $\chi=(u) v, \mathscr{M}_{E}(x) \times \mathscr{M}(x, y)$ so that for some $\rho \in \mathbb{R}$, we have $u(\tau)=\exp _{x} \xi(\tau)$ for $\tau \geqq \rho$ and $v(\tau)=\exp _{x} \zeta(\tau)$ for $\tau \leqq \rho$, we define $w_{\chi}=u \hat{\#} v \in \mathscr{P}(y)$ by $w_{\chi}=v$ on $S_{\rho-1}$ and $w_{\chi}=u$ on $S-S_{\rho+1}$ and $w_{\chi}(\tau)=$ $\exp _{x}(\beta(\tau-\rho) \zeta(\tau)+\beta(-\tau-\rho) \xi(\tau))$ on $S_{\rho+1}-S_{\rho-1}$.

Lemma 2e.3. Let $E \# E^{\prime}$ be defined by (2d.3). Then we have a differentiable map

$$
\begin{gathered}
\# \mathscr{M}_{E}(x ; E) \times \mathscr{M}_{E^{\prime}}(x, y) \rightarrow \mathscr{M}_{E \# E^{\prime}}(y), \\
\chi=\left(u^{\prime}, v\right) \mid \rightarrow \exp _{w_{\chi}} \xi,
\end{gathered}
$$

for some open set $U$ of $\mathscr{M}(x, y)$. Moreover, we can choose so that $\|\xi\|_{1, p}$ becomes arbitrarily small.

Proof. As in the proof of Proposition 2d, we apply Picard's method (Lemma 2d.1) to the map $\pi^{\perp} \bar{\partial}_{J, H}\left[w_{\chi}\right]$, where $\pi^{\perp}$ is the $L^{2}$-orthogonal projection onto the complement of $E \widehat{\# E}$.

Now we have as an immediate consequence: 
Proposition 2e.1. For each $u \in \mathscr{M}(x, y)$, define the orientation of $\mathscr{M}(x, y)$ near $u$ as the unique orientation for which the map (5) is orientation preserving. Then (1) is orientation preserving.

\section{Compactness}

3a. Weak Topologies. Let $\theta$ be any surface with or without boundary which is equipped with a metric. Then for any map $u \in C^{r}(\theta, P)$, define

$$
\mathcal{O}_{r, \varepsilon}(u)=\left\{\exp _{u} \xi \mid \xi \in C^{r}\left(u^{*} T P\right) \text { and }\|\xi\|_{r, \infty}<\varepsilon\right\} \text {. }
$$

The exponential function exp is defined in Sect. 2a. If $w: \theta \supset \theta^{\prime} \rightarrow P$ is another $C^{r}$-function defined on a subset of $\theta$, then we will also say that $w \in \mathcal{O}_{r, \varepsilon}(u)$ if $w \in \mathcal{O}_{r, \varepsilon}\left(\left.u\right|_{\theta^{\prime}}\right)$. The domain of a map is always clear from the context. If $\theta=\mathbb{R} \times S^{1}$ and $u \in \mathscr{P}(x, y)$, then $\mathcal{O}(u)$ is a neighborhood of $u$ in $\mathscr{P}(x, y)$. We want to define certain "neighborhoods of infinity" in $\mathscr{P}(x, y)$. More precisely, our first aim is to formalize the notion of "geometric convergence" of trajectory to a $k$-trajectory.

Definition 3a.1. The $\varepsilon$-neighborhood $\mathcal{O}_{r, \varepsilon}(\underline{u})$ of $u \in \mathscr{M}^{k}(x, y)$ in $\mathscr{P}(x, y)$ is the set of all $\omega \in \mathscr{P}(x, y)$ such that there exists a covering

$$
\mathbb{R}=\bigcup_{x \in x} I_{x} \cup \bigcup_{u \in u} I_{u}
$$

with the following property. There exists real numbers $\tau_{x} \in I_{x}$ and $\tau_{u}=I_{u}$ such that if $w_{x}\left(w_{u}\right)$ are obtained from $w$ by restricting $w$ to $I_{x}\left(I_{u}\right)$ and translating by $\tau_{x}\left(\tau_{u}\right)$, then $w_{x} \in \mathcal{O}_{r, \varepsilon}(\hat{x})$ and $w_{u} \in \mathcal{O}_{r, \varepsilon}(u)$.

There are several stronger and weaker versions of convergence, which all turn out to be equivalent for elements of $\hat{\mathscr{M}}(x, y)$. Clearly, the families $u_{\alpha}:=\#\left(\underline{u}, \rho_{\alpha}\right)$ of Proposition $1 \mathrm{~b}$ converge to $\underline{u}$ in the sense of Definition 3a.1 if and only if $\rho_{k \alpha} \rightarrow \infty$ for all $k$. One can show (see Proposition $3 c$ below) that if $\underline{u} \in \mathscr{M}^{k}(x, y)$ is nondegenerate, then any sequence $u_{\alpha}$ converging to $\underline{u}$ is ultimately contained in the image of the map \# of Proposition 1b. Moreover, from finite dimensional Morse theory, we would expect that $\hat{\mathscr{M}}$ is compact under the convergence of Definition $3 \mathrm{a} .1$ as long as $\mathscr{S}$ is compact in $\Omega$. This is in fact the case, as we will see later in this section. If we only assume $\mathscr{S}$ to be bounded, we need a weaker notion of convergence. First, we extend $\mathscr{M}^{k}$ as follows:

Definition $3 a .2$. A cusp trajectory $(\underline{u}, \underline{v}) \in \hat{\mathscr{M}} \mathscr{C}^{k}(x, y)$ is a $k$-trajectory $u \in \hat{\mathscr{M}}^{k}(x, y)$ together with a finite collection $v$ holomorphic maps $v: \mathbb{C} \rightarrow(P, J)$ of finite area $\|D v\|_{2}$ labeled by an element $w_{v} \in \underline{x} \cup \underline{u} \cup \underline{v}$ and a point $\theta_{v}$ in $\mathbb{R} \times S^{1}$ or $S^{2}$ such that

$$
v_{\infty}:=\lim _{|z| \rightarrow \infty} v(z)=w_{v}\left(\theta_{v}\right)
$$

By "removing the singularity" (see $[\mathrm{G}]$ and $[\mathrm{P}]$ ), each $v \in \underline{v}$ is obtained from a holomorphic map $\tilde{v}: S^{2} \rightarrow P$ by a conformal equivalence. The points in $P$ defined by (2) are called cusp points. Again, there are many different possible neighborhood systems which are all equivalent by elliptic regularity. In particular, since locally, 
$(P, J)$ approaches the standard complex space $\mathbb{C}^{n}$, the behavior of holomorphic curves is rather predictable in the neighborhoods of the cusp curves. This motivates the following approach to "weak topology":

Definition 3a.3. The weak $\varepsilon$-neighborhood $\mathcal{O}_{r, \varepsilon}(\underline{u}, \underline{v}) \quad$ of $\quad(\underline{u}, \underline{v}) \in \hat{\mathscr{M}} \mathscr{C}(x, y)$ in $\mathscr{P}(x, y)$ is the set of all $w \in \mathscr{P}(x, y)$ such that

$$
Z=w^{-1}\left[P-\bigcup_{v \in 1} B_{\varepsilon}\left(v_{\infty}\right)\right] \in \mathbb{R} \times S^{1}
$$

decomposes into one ubounded component $Z_{0}$ and one bounded component $Z_{v}$ for each $v \in \underline{v}$ such that

(W1) $\left.w\right|_{Z_{0} \in \mathcal{O}_{r, \varepsilon}}(\underline{u})$

(W2) A suitable dilation $w^{\circ} \delta_{v}$ of $w$ on $Z_{v}$ is contained on $\mathcal{O}_{r, \varepsilon}(v)$,

(W3) $\delta_{v}(\theta(v)) \in Z_{v}$.

Note that Definition 3a.3 (as opposed to Definition 3a.1) relies essentially on the "double nature" of $\mathscr{P}$ as a space of paths in $\Omega$ and a space of 2-dimensional maps into $P$. The inverse images of $B_{\varepsilon}\left(v_{\infty}\right)$ in $\mathbb{R} \times S^{1}$ correspond to neighborhoods of Gromov's "small geodesics," which decompose the Riemann surface $\Sigma$ in the general compactness theory of holomorphic maps $\Sigma \rightarrow(P, J)$. Condition (W3) keeps track of "hierarchy" of cusp points represented by labels. It is not essential in an application and we might have omitted it along with the labels $(w, \theta)$, except that a "purely geometric" definition of $\hat{M} \mathscr{C}$ might allow for confusingly many different components in $\mathcal{O}_{r, \varepsilon}(\underline{u}, \underline{v})$.

3b. Local Convergence. For any interval $I \subset \mathbb{R}$, we define $\mathscr{P}_{k \mid I}^{p}=L_{1}^{p}\left(I \times S^{1}, P\right)$. We also write $\mathscr{P}_{k \mid \alpha}^{p}=\mathscr{P}_{k \mid[-\alpha, \alpha]}^{p}$. The purpose of this section is to prove the following result:

Proposition 3b. Let $u_{\alpha} \in \mathscr{P}_{k \mid \alpha}^{p}, \alpha \in \mathbb{N}$, be a sequence which takes values in some compact subset of $P$. Assume that $l\left(u_{\alpha}\right)$ is bounded and that $J_{\alpha}, H_{\alpha} \rightarrow J, H$ is a convergent sequence in $\mathscr{J} / \mathscr{H}$ such that $\lim _{\alpha \rightarrow \infty}\left\|\bar{\partial}_{J_{x}, H_{\alpha}} u_{\alpha}\right\|_{k, p}=0$. Then there exists a subsequence $u_{\alpha}$ converging in $L_{k+1}^{p}$ to a cusp trajectory $(u, \underline{v}) \in \mathscr{M} \mathscr{C}_{J, H}$. We have

$$
\sum_{i=1}^{N}\left\langle\omega, v_{i}\right\rangle+l^{2}(u) \leqq \lim _{\alpha \rightarrow \infty} \sup l^{2}\left(u_{\alpha}\right)
$$

Note in particular that if $l^{2}\left(u_{\alpha}\right) \leqq l$ for some $l$ smaller than

$$
l_{0}=\min \left\{\langle\omega, v\rangle \mid v \in H_{2}(P, \mathbb{Z}) \text { and }\langle\omega, v\rangle>0\right\},
$$

then (1) implies that the set $\underline{v}$ is empty, i.e. that $u_{\rho}$ converges locally in $L_{k}^{p}$ to $u$.

Proof of Proposition $3 b$. The central ingredient of the proof is the following standard result of elliptic regularity theory on the Cauchy Riemann operator. 
Lemma 3b.1. ([F4], Lemma 2.3). Let $U$ be an open ball in $\mathbb{C}$ and let $J$ be a smooth almost complex structure on $P$. Then

(1) For any compact set $K \subset U$ and $l \in \mathbb{N}$ there exists a continuous function $C: \mathbb{R}_{+}^{2} \rightarrow \mathbb{R}_{+}$so that

$$
\|u\|_{l, p} \leqq C\left(\left\|\bar{\partial}_{J} u\right\|_{l-1, p},\|u\|_{1, p}\right) .
$$

(2) If $u_{\alpha}$ is a sequence in $L_{1}^{p}(U, P)$ with $\lim _{\alpha \rightarrow \infty}\left\|\bar{\partial}_{J} u\right\|_{l-1, p}=0$ and if $\left\|u_{\alpha}\right\|_{1, p}$ is bounded, then there exists a subsequence $u_{\alpha}$ converging in $L_{l}^{p}(K, P)$ to some holomorphic map $u: K \rightarrow P$.

A related result is

Lemma 3b.2. For every open $I \subset \mathbb{R}$ and for every bounded sequence $\left\{u_{\rho}\right\}$ in $\left.\mathscr{P}\right|_{I}$ satisfying

$$
\left\|\bar{\partial}_{J_{\alpha}, H_{\alpha}}\left(u_{\alpha}\right)\right\|_{p} \rightarrow 0
$$

with $\left(J_{\rho}, H_{\rho}\right) \rightarrow(J, H)$ in $\mathscr{J} \times \mathscr{H}$, there exists a subsequence which converges in $\mathscr{P}_{\mid K}$ for compact $K \subset I$ to a solution $u$ of $\bar{\partial}_{J, H} u=0$.

Now consider as in $[\mathrm{F} 4]$ the sequence of numbers

$$
\varepsilon_{\alpha}(K):=\inf \left\{\varepsilon>0 \mid \text { there exists } \theta \in K \times S^{1} \text { so that }\left\|D u_{\alpha}\right\|_{p, B_{\varepsilon}(\theta)}=\varepsilon^{2 / p}\right\} .
$$

If $\varepsilon_{\alpha}$ is bounded away from zero for some compact interval $K \subset \mathbb{R}$, then the hypothesis of Lemma 3b.2 is satisfied, since $\left\|D u_{\alpha \mid K}\right\|_{p}$ is bounded. Hence assume that $\varepsilon_{\alpha} \rightarrow 0$ for a subsequence and choose $\theta_{\alpha} \in K \times S^{1}$ so that $\left\|D u_{\alpha}\right\|_{p, B_{\alpha}} \geqq \frac{1}{2} \varepsilon_{\alpha}^{2 / p}$ for $B_{\alpha}=B_{\varepsilon_{\alpha}}\left(\theta_{\alpha}\right)$. Then for any $R>0$ and for $\alpha$ large enough we have maps

$$
\begin{gathered}
v_{\alpha}: \mathbb{C} \supset B_{R}(0) \rightarrow P, \\
v_{\alpha}(\theta)=u_{\alpha}\left(\varepsilon_{\alpha} \cdot\left(\theta-\theta_{\alpha}\right)\right),
\end{gathered}
$$

satisfying

$$
\begin{aligned}
& \left\|D v_{\alpha}\right\|_{2}^{2} \leqq a, \\
& \left\|D v_{\alpha}\right\|_{p, B_{1}(0)} \geqq \frac{1}{2}, \\
& \left\|D v_{\alpha}\right\|_{p, B_{1}(\theta)} \leqq 1 \text { for all } \theta \in B_{R-1}(0), \\
& \left\|\bar{\partial} v_{\alpha}-\varepsilon_{\alpha}^{*} \nabla H_{\alpha}\left(u_{\alpha}\right)\right\|_{p} \rightarrow 0 .
\end{aligned}
$$

In (4) we have use the fact that $\|D u\|_{2}$ is conformally invariant and that therefore

$$
\left\|D v_{\alpha}\right\|_{2}^{2} \leqq\left\|D u_{\alpha \mid B_{\varepsilon_{\alpha}}}\right\|_{2}^{2} \leqq l^{2}\left(u_{\alpha}\right)+\varepsilon_{\alpha}\left\|H_{\alpha}\right\|_{1, \alpha},
$$

by the definition of $l^{2}$ in (1b.4). Equations (5) and (6) are immediate consequences of the rescaling. To prove (7), we calculate

$$
\bar{\partial} v_{\alpha}(\tau, t)=\varepsilon_{\alpha} \bar{\partial} u_{\alpha}\left(\varepsilon_{\alpha}\left((\tau, t)-\left(\tau_{\alpha}, t_{\alpha}\right)\right)\right)=\varepsilon_{\alpha} \cdot \pi_{\alpha}\left(u_{\alpha}\left(\varepsilon_{\alpha}\left(\tau-\tau_{\alpha}\right)\right)\right)\left(\varepsilon_{\alpha}\left(t-t_{\alpha}\right)\right),
$$

so that

$$
\begin{aligned}
\left\|\bar{\partial} v_{\alpha}\right\|_{p}^{p} & \leqq \varepsilon_{\alpha}^{p} \int_{B_{R}}\left|H_{\alpha}\left(u_{\alpha}\left(\varepsilon_{\alpha}\left(\tau-\tau_{\alpha}\right)\right)\right)\left(\varepsilon_{\alpha}\left(t-t_{\alpha}\right)\right)\right|^{p} d t d \tau \\
& \leqq \varepsilon_{\alpha}^{p-1} \int_{-R}^{R}\left\|H_{\alpha}\left(u_{\alpha}\left(\varepsilon_{\alpha}\left(\tau-\tau_{\alpha}\right)\right)\right)\right\|_{p}^{p} d \tau \leqq C \varepsilon_{\alpha}^{p-2}\left\|H_{\alpha}\right\|_{1, \infty}
\end{aligned}
$$


converges to zero for $p>2$. Now it follows from Lemma 3a.1 that a subsequence $v_{\alpha}$ converges for any $R$ in $L_{1}^{p}\left(B_{R}, P\right)$ to some holomorphic map $v: \mathbb{C} \rightarrow P$ satisfying $\|D v\|_{2}^{2} \leqq a$ and $\|D v\|_{p, B_{1}(0)} \geqq \frac{1}{2}$. The second property implies that $v$ is nonconstant, whereas the first implies by (see $[\mathrm{Pu}]$ ) that there exists a holomorphic map $\bar{v}: S^{2} \rightarrow P$ so that $v=\bar{v}^{\circ} \gamma$, where $\gamma: \mathbb{C} \rightarrow \mathscr{S}^{2}$ is the stereographic projection. It follows in particular that $\|D v\|_{2}^{2}$ is bounded from below by a positive constant depending only on the (finitely generated) homology group $\mathrm{H}_{2}(P)$ and on $\omega$.

We now repeat this procedure on $K_{\alpha}=\mathbb{R} \times S^{1}-\bar{U}_{\alpha}$ with $\bar{U}_{\alpha}=B_{\sqrt{\varepsilon_{\alpha}}}(\theta)$. That is, we consider the numbers $\varepsilon_{\sigma}^{\prime}$ defined by $(E K)$ with $\bar{U}_{\alpha}$ removed from $K$. If $\varepsilon_{\alpha}^{\prime} \rightarrow 0$, then the corresponding reparametrized sequence $v_{\alpha}^{\prime}$ converges as before to a nontrivial holomorphic map $S^{2} \rightarrow P$. To see that $\lim \sup l^{2}\left(u_{\alpha}\right)$ must be at least the sum of the actions of $v$ and $v^{\prime}$, note that $\theta_{\alpha}^{\prime}$ tends to infinity in the reparametrized chart around $\theta_{\alpha}$, since $\operatorname{dist}\left(\theta_{\alpha}, \theta_{\alpha}^{\prime}\right) \geqq \varepsilon_{\alpha}^{1 / 2}$. If also $\operatorname{dist}\left(\theta_{\alpha}, \theta_{\alpha}^{\prime}\right) / \varepsilon_{\alpha}^{\prime} \rightarrow \infty$, then the converse is also true, so that there exist disjoint neighborhoods $V_{\alpha}$ of $\theta_{\alpha}$ and $V_{\alpha}^{\prime}$ of $\theta_{\alpha}^{\prime}$ so that $\left\|D u_{\alpha \mid v_{\alpha}}\right\|_{2}^{2}$ and $\left\|D u_{\alpha \mid V_{\alpha}^{\prime}}\right\|_{2}^{2}$ approach the actions of $v$ and $v^{\prime}$, respectively. On the other hand, if $\operatorname{dist}\left(\theta_{\alpha}, \theta_{\alpha}^{\prime}\right) / \varepsilon_{\alpha}^{\prime}$ is bounded, the $\varepsilon_{\alpha}^{\prime} / \varepsilon_{\alpha} \rightarrow \infty$, and we can choose $V_{\alpha}$ and $V_{\alpha}^{\prime}$ so that $\left\|D u_{\alpha \mid V_{\alpha}}\right\|_{2}^{2}$ and $\left\|D u_{\alpha \mid V_{\alpha}^{\prime}-V_{\alpha}}\right\|_{2}^{2}$ approach the actions of $v$ and $v^{\prime}$. Either way, we conclude that $l^{2} \geqq\langle\omega, v\rangle+\left\langle\omega, v^{\prime}\right\rangle$. Since $\langle\omega, v\rangle \geqq l_{0}$ for any nonconstant holomorphic curve, this process terminates after finitely many steps. This completes the proof of Proposition $3 \mathrm{~b}$.

3c. Global Convergence. In this section, we conclude the proof of Proposition $1 \mathrm{~b}$ by proving

Proposition 3c. (1) For each $u \in \mathscr{M}_{J \pi}$ there exist $x, y \in \mathscr{Z}_{J \pi}$ such that $\lim _{\tau \rightarrow-\infty} u(\tau)=x$ and $\lim _{\tau \rightarrow \infty} u(\tau)=y$ in $\Omega$. In fact, if $x$ and $y$ are nondegenerate, then the set of such trajectories with the local topology is homeomorphic to $\mathscr{M}(x, y)$.

(2) Let $\left(J_{\alpha}, H_{a}\right) \rightarrow(J, H)$ be convergent sequences in $\mathscr{J} \times \mathscr{H}$ and let $\left(x_{\alpha}, y_{\alpha}\right) \in$ $\mathscr{Z}_{J_{\alpha}, H_{\alpha}}^{2}$ converge to $(x, y) \in \mathscr{Z}_{H}^{2}$ in $\Omega$. Then for any sequence $u_{\alpha} \in \mathscr{M}_{J_{\alpha} H_{\alpha}}\left(x_{\alpha}, y_{\alpha}\right)$ with constant index Index $\left(u_{\alpha}\right)=I$, there exists a subsequence converging to some $(\underline{v}, \underline{w}) \in \mathscr{M} \mathscr{C}_{J, H}^{k}(x, y)$ for some $k \geqq 0$. Moreover, we have

$$
\begin{aligned}
I & =\sum_{i} \operatorname{Index}\left(v_{i}\right)+2 \sum_{j} c_{1}\left(w_{j}\right), \\
l^{2}\left(u_{\alpha}\right) & =\sum_{i} l^{2}\left(v_{i}\right)+\sum_{j}\left\|D w_{j}\right\|_{2}^{2} .
\end{aligned}
$$

If $\underline{w}$ is trivial, i.e. if $u_{\alpha}$ converges to $\underline{v} \in \mathscr{M}_{J \pi}^{k}$, then for every $\varepsilon>0$ there exists an $N>0$ so that for $\rho>N, u_{\alpha}$ is contained in the $\varepsilon$-tube

$$
\mathscr{U}_{\varepsilon}(\underline{v})=\left\{\exp _{v_{\mathfrak{\imath}}(\tau)}(\xi) \mid\|\xi\|_{\infty}<\varepsilon, 1 \leqq i \leqq k \text {, and } \tau \in \mathbb{R}\right\}
$$

If $\underline{v} \in \hat{\mathscr{M}}^{k}(x, y)$ is regular, then there exists $\varepsilon>0$ so that all trajectories in $\hat{\mathscr{M}}(x, y)$ which are fully contained in $\mathscr{U}_{\varepsilon}(\underline{v})$ are in the image of the map \# of Proposition 2d. 
Proof of Proposition 3c. (1) To prove the first assertion, we assume the contrary, i.e. we assume that there exists a sequence $\tau_{\alpha} \rightarrow \infty$ so that $u\left(\tau_{\alpha}\right)$ does not accumulate at $\mathscr{Z}$. Then the sequence $u_{\alpha}(\tau)=u\left(\tau-\tau_{\alpha}\right)$ on $\left[-\tau_{\alpha} / 2, \tau_{\alpha} / 2\right]$ satisfies the hypothesis of Proposition $3 \mathrm{~b}$ with $\lim _{p \rightarrow \infty} l\left(u_{\alpha}\right) \rightarrow 0$. Hence $u_{\alpha}$ converges locally to some constant trajectory, which contradicts the above assumption.

In the same way we prove that for every $\varepsilon>0$ there exists $r>0$ so that $u=\exp _{x} \xi$ on $[r, \infty)$ with $\left\|\xi_{[[\rho, \rho+1]}\right\|_{1, p}<\varepsilon$ for all $\rho \geqq r$. To prove exponential decay, we proceed in a similar way as in the proof of Theorem 4 of [F4]: Define $\beta_{\sigma}(\tau)=$ $\beta(\tau-\sigma+1) \beta(-\tau+\sigma)$ and consider the function $f(\sigma)=\left\|\beta_{\sigma} \xi\right\|_{2}^{2}$. Then if for some $\mu>0$, we have

$$
f^{\prime \prime}(\sigma) \geqq \mu f(\sigma),
$$

it follows e.g. from the maximum principle that $f$ decays exponentially. To prove (1), we calculate with $g(\xi)=g_{J \pi}[x](\xi)$

$$
\begin{aligned}
f^{\prime \prime}(\tau) & =\int \beta^{2}(\tau)\left\{\left\|\xi^{\prime}(\tau)\right\|_{2}^{2}+\left\langle\xi(\tau), \xi^{\prime \prime}(\tau)\right\rangle\right\} d \tau \\
& =\int \beta^{2}(\tau)\left\{\|g(\xi(\tau))\|_{2}^{2}+\langle\xi(\tau), D g(\xi(\tau)) g(\xi(\tau))\rangle\right\} d \tau .
\end{aligned}
$$

To estimate the second term, note that with $D_{\tau}=D_{g}(\xi(\tau))$ and expanding $g$ around $x$,

$$
\left\langle\xi(\tau), D_{\tau} g(\xi(\tau))\right\rangle=\left\langle D_{\tau}^{+} \xi(\tau), D_{\tau} \xi(\tau)\right\rangle+\left\langle D_{\tau}^{+} \xi(\tau), N(\xi(\tau))\right\rangle,
$$

where $N$ is the nonlinear term in the first order expansion of $g$ around $x$. Since $D_{\tau}$ has no purely imaginary eigenvalues, the first term is positive. The nonlinear term can be estimated as follows,

$$
\begin{aligned}
\left\langle D_{\tau}^{+} \xi(\tau), N(\xi(\tau))\right\rangle & \leqq C\left\|D_{\tau}^{+} \xi(\tau)\right\|_{2}\|\xi(\tau)\|_{\infty}\|\xi(\tau)\|_{1,2} \\
& \leqq \varepsilon(r)\|\xi(\tau)\|_{1,2}^{2}
\end{aligned}
$$

with $\lim _{r \rightarrow \infty} \varepsilon(r)=0$. Since $x$ is nondegenerate zero of $g$, we have $\|g(\xi)\|_{2} \geqq$ $\gamma\|\xi\|_{1,2} \geqq \gamma\|\xi\|_{1,2}$ for some $\gamma>0$. Hence for $\tau$ large enough, the nonlinear term is small compared with the first term of (2). This implies (1) and hence exponential decay of $f(\tau)$.

Using (2b.3) we can improve this result to conclude that $\lim _{\sigma \rightarrow \infty}\left\|\beta_{\sigma} \xi\right\|_{1, p}=0$. Now define $\beta_{r R}(\tau)=\beta(-\tau-r) \beta(\tau-R)$ and consider the expansion (2a.3) around $\hat{x}$,

$$
\bar{\partial}[\hat{x}]\left(\beta_{r R} \xi\right)=D_{\hat{x}}\left(\beta_{r R} \xi\right)+N\left(\beta_{r R} \xi\right) .
$$

Then by (2a.5) and (2b.3),

$$
\begin{aligned}
\left\|\beta_{r R} \xi\right\|_{1, p, \delta} & \leqq C\left\|D_{\chi} \beta_{r R} \xi\right\|_{p} \leqq C\left\|N\left(\beta_{r R} \xi\right)\right\|_{p}+\left\|\bar{\partial}_{J \pi}\left(\exp _{x}\left(\beta_{r R} \xi\right)\right)\right\|_{p} \\
& \leqq \varepsilon(r)\left\|\beta_{r R} \xi\right\|_{1, p}+C,
\end{aligned}
$$

with $\lim _{n \rightarrow \infty} \varepsilon(r)=0$ by the above. For $r$ large enough, this yields an estimate on $\left\|\beta_{r R} \xi\right\|_{1, p ; \delta}^{n \rightarrow \infty}$ which does not depend on $R$. This proves that $u \in \mathscr{P}(x, y)$. The same 
method can be used to compare the topology on $\mathscr{P}(x, y)$ with the local topology on $\mathscr{M}(x, y)$.

Proof of Proposition 3c. (2) The first assertion follows from a simple iterative argument applying (1) and Proposition $3 \mathrm{~b}$ to suitably rescaled sequences. The index formula follows from (2b.1) and (2b.2). The last assertion is proved indirectly: Assume that there exists $\varepsilon>0$ and subsequence $u_{\alpha}$ such that $u_{\alpha}\left(\tau_{\alpha}\right) \notin \mathscr{U}_{\varepsilon}(\underline{v})$ for some $\tau_{\alpha} \in \mathbb{R}$. Then by Proposition $3 b$, there exists a subsequence converging to some holomorphic curve which is not identical with any of the $v_{i}$, in contradiction to the assumption. For more details, see [F4], Sect. 2.

It remains to prove that for regular $\underline{v}$ and $\varepsilon$ small enough, each $w \in \mathscr{M}(x, y)$ which is contained in $\mathscr{U}_{\varepsilon}(\underline{v})$ is in the image of \#. By the uniqueness property of Picard's method, it suffices to prove that it is $L_{1}^{4}$-close to the image of $\hat{\#}$. Define therefore numbers $s_{i}$ so that $a\left(w\left(s_{i}\right)\right)=a\left(y_{i}\right)$ for $0<i<k$. By Proposition $3 \mathrm{~b}$, we have for $\varepsilon$ small enough $w=\exp _{y_{i}}(\eta)$ on $I_{i}=\left[s_{i}-1, s_{i}+1\right]$ with

$$
\left\|\eta_{\mid I_{l}}\right\|_{1, p} \leqq \phi_{1}(\varepsilon)
$$

Here and in the following, $\phi_{\alpha}: \mathbb{R} \rightarrow \mathbb{R}$ denotes a continuous map which is independent of $w$ and satisfies $\phi_{\alpha}(0)=0$. For $1 \leqq i \leqq k$, we can define $\hat{v}_{i} \in \mathscr{P}\left(y_{i-1}, y_{i}\right)$ by

$$
\hat{v}_{i}(\tau) \begin{cases}\exp _{y_{t}-1} \beta\left(\tau-s_{i-1}\right) \xi(\tau) & \text { for } \tau \leqq s_{i-1}+1 \\ \exp _{y_{i}} \beta\left(-\tau-s_{i}\right) \xi(\tau) & \text { for } \tau \geqq s_{i}-1 \\ u(\tau) & \text { otherwise }\end{cases}
$$

Now define $\tau_{i} \in \mathbb{R}$ by

$$
a\left(w\left(\tau_{i}\right)\right)=\frac{1}{2}\left(a\left(y_{i-1}\right)+a\left(y_{i}\right)\right) .
$$

Then for $\hat{\chi}=\left(\tau_{i} * \hat{v}_{i} ; \frac{1}{2}\left(s_{i}+s_{i+1}\right)\right)$, we have $w=\exp _{w_{\hat{\gamma}}} \hat{\xi}$ with $\|\hat{\xi}\|_{1, p} \leqq \phi_{2}(\varepsilon)$, so that it remains to show that each $\tau_{i *} \hat{u}_{i}$ is close enough to $u_{i}$. (Here, we identify $u \in \hat{\mathscr{M}}(x, y) \subset \mathscr{M}(x, y)$ by the condition $a(u(0)) \frac{1}{2}(a(x)+a(y))$.) From Proposition $3 \mathrm{~b}$ it follows again that $\tau_{i} * \hat{u}_{i}=\exp _{w} \xi_{i}$ with $\left\|\xi_{i}\right\|_{1, \infty} \leqq \phi_{3}(\varepsilon)$. To obtain $L^{p}$-estimates, we expand as in (3a.3)

$$
\bar{\partial}\left[v_{i}\right]\left(\xi_{i}\right)=D_{v_{i}} \xi_{i}+N_{v_{i}}\left(\xi_{i}\right)
$$

Since $v_{i}$ is regular and since on the finite dimensional kernel of $D_{v_{\mathrm{t}}}$ all norms are equivalent, we have

$$
\begin{aligned}
\left\|\xi_{i}\right\|_{1, p} & \leqq C\left(\left\|D_{v_{i}} \xi_{i}\right\|_{p}+\|\xi\|_{\infty}\right) \leqq C\left(\left\|\bar{\partial}_{v_{i}}\left(\xi_{i}\right)-N_{v_{i}}\left(\xi_{i}\right)\right\|_{p}+\|\xi\|_{\infty}\right) \\
& \leqq C\left(\left\|\bar{\partial} \hat{v}_{i}\right\|_{p}+\left\|N_{v_{i}}\left(\xi_{i}\right)\right\|_{p}\right)+\phi_{3}(\varepsilon) .
\end{aligned}
$$

Note that $\left\|\xi_{i}\right\|_{p}<\infty$, since $v_{i}, \hat{v}_{i} \in \mathscr{P}\left(x_{i-1}, x_{i}\right)$. Now we have $\left\|\bar{\partial} \hat{v}_{i}\right\|_{p} \leqq \phi_{4}(\varepsilon)$ by (3c.3). Moreover, by (2a.4),

$$
\left\|N_{v_{i}}\left(\xi_{i}\right)\right\|_{p} \leqq C(v)\left\|\xi_{i}\right\|_{1, \infty}\left\|\xi_{i}\right\|_{p} \leqq \phi_{5}(\varepsilon)\left\|\xi_{i}\right\|_{p} .
$$

This together with the previous estimate proves that $\lim _{\varepsilon \rightarrow 0}\left\|\xi_{i}\right\|_{1, p}=0$ for all $i$. This completes the proof of Proposition 3c. 
Acknowledgements. The present work owes much to the ideas of and valuable discussions with C. Conley, M. Gromov, and E. Zehnder. Moreover, many analytic details in Sects. 2 and 3 contain ideas which are due to C. Taubes. I would also like to thank M. Atiyah, H. Hofer, D. McDuff, and A. Weinstein for general discussions on the subject.

\section{References}

[A1] Arnold, V. I.: Sur une propriete topologique des applications globalement canoniques de la mecanique classique, C. R. Acad. Sci. Paris 261, 3719-3722 (1965)

[A2] - Mathematical methods of classical mechanics (Appendix 9), Nauka 1974; Engl. Transl. Berlin, Heidelberg, New York: Springer 1978

[An] Aronszajn, N.: A unique continuation theorem for solutions of elliptic partial differential equations or inequalities of the second order. J. Math. Pures Appl. 36, 235-249 (1957)

[AS1] Atiyah, M. F., Singer, I. M.: The index of elliptic operators I, III. Ann. Math. 87, 484-530 (1968); 87, 546-604 (1968)

[AS2] — - Index theory of skew adjoint Fredholm operators. Publ. Math. IHES 37, 305-325 (1969)

[B] Banyaga, - .: Sur la groupe des diffeomorphismes qui preservent une forme symplectique. Comment. Math. Helv. 53, 174-227 (1978)

[Bi] Birkhoff, G. D.: Proof of Poincaré's geometric theorem. Trans. AMS 14, 14-22 (1912)

[Ch] Chaperon, M.: Quelques questions de geometrie symplectique [d'apres, entre autres, Poincaré, Arnold, Conley et Zehnder], Seminaire Bourbaki 1982-83. Asterisque 105-106, 231-249 (1983)

[C] Conley, C. C.: Isolated invariant sets and the Morse index, CBMS Reg. Conf. Series in Math 38. Providence, RI: AMS 1978

[CZ1] Conley, C. C., Zehnder, E.: The Birkhoff-Lewis fixed point theorem and a conjecture of V. I. Arnold. Invent. Math. 73, 33-49 (1983)

[CZ2] — - Morse type index theory for flows and periodic solutions for Hamiltonian equations. Commun. Pure and Appl. Math. 34, 207-253 (1984)

[CZ3] — - : Subharmonic solutions and Morse-theory. Physica 124A, 649-658 (1984)

[E] Eliashberg, Y. M.: Estimates on the number of fixed points of area preserving mappings. Preprint, : Syktyvkar University 1978

[FHV] Floer, A., Hofer, H., Viterbo, C.: The Weinstein conjecture on $P \times \mathbb{C}$, preprint

[F1] Floer, A.: Proof of the Arnold conjecture for surfaces and generalizations to certain Kähler manifolds. Duke Math. J. 53, 1-32 (1986)

[F2] - A refinement of the Conley index and an application to the stability of hyperbolic invariant sets. Ergod. Theoret Dyn. Sys. 7, 93-103 (1987)

[F3] - : Morse theory for Lagrangian intersections. J. Diff. Geom. 28, (1988)

[F4] - The unregularized gradient flow of the symplectic action. Commun. Pure Appl. Math. (to appear)

[F5] — : A relative Morse index for the symplectic action. Commun. Pure Appl. Math. (to appear)

[F6] - Witten's complex and infinite dimensional Morse theory. J. Diff. Geom. (to appear)

[F7] — : Cuplength estimates for Lagrangian intersections (to appear)

[F8] - An instanton-invariant for 3-manifolds. Commun. Math. Phys. 118, 215-240 (1988)

[Fo] Fortune, B.: A symplectic fixed point theorem for $\mathrm{CP}^{n}$. Invent. Math. 81, 29-46 (1985)

[Fr] Frnsoza, R.: Index filtrations and connection matrices for partially ordered Morse decompositions. Preprint

[G] Gromov, M.: Pseudo-holomorphic curves in symplectic manifolds. Invent. Math. 82, 307-347 (1985)

[Ho1] Hofer, H.: Lagrangian embeddings and critical point theory. Ann. Inst. H. Poincaré. Analyse non lineaire 2, 407-462 (1985)

[HoZ] Hofer, H.: Ljusternik-Snirelman theory for Lagrangian intersections, preprint

[H] Hörmander, L.: The analysis of linear differential operators III. Berlin, Heidelberg, New York: Springer 1985

[Hu] Husemoller, D.: Fibre bundles, Springer Grad. Texts in Math. vol. 20. Berlin, Heidelberg, New York: Springer 1966 
[Ka] Kawabe, H.: A symplectic fixed point theorem in case that a symplectic form is exact in the universal covering space and a manifold has no conjugate points, Preprint, Tokyo Inst. of Tech. 1987

[K1] Klingenberg, W.: Lectures on closed geodesics. Grundl. der math. Wiss vol. 230. Berlin, Heidelberg, New York: Springer 1978

[Ko] Kondrat'ev, V. A.: Boundary value problems for elliptic equations in domains with conical or angular point. Trans. Mosc. Math. Soc. 16, (1967)

$[\mathrm{Ku}]$ Kuiper, N. H.: The homotopy type of the unitary group of Hilbert space. Topology 3, 19-30 (1965)

[LS] Laudenbach, F., Sikorav, J. C.: Persistance d'intersection avec la section nulle au cours d'une isotopie hamiltonienne dans un fibre cotangent. Invent. Math. 82, 349-357 (1985)

[LM] Lockhard, R. B., McOwen, R. C.: Elliptic operators on noncompact manifolds. Ann. Sci. Norm. Sup. Pisa IV-12, 409-446 (1985)

[MW] Marsden, J., Weinstein, A.: Reduction of symplectic manifolds with symmetry. Rep. Math. Phys. 5, 121-130 (1974)

[MP] Mazja, V. G., Plamenevski, B. A.: Estimates on $L^{p}$ and Hölder classes and the Miranda-Agmon maximum principle for solutions of elliptic boundary problems in domans with singular points on the boundary. Math. Nachr. 81, 25-82 (1978) (in Russian), English Transl. In: AMS Trans., Ser. 2, 123, 1-56 (1984)

[McD] McDuff, D.: Examples of symplectic structures. Invent. Math. 89, 13-36 (1987)

[M] Milnor, J.: Lectures on the h-cobordism theorem. Math. Notes, Princeton, NJ: Princeton Univ. Press 1965

[N] Nikishin, N.: Fixed points of diffeomorphisms on the two sphere that preserve area. Funk. Anal. i Prel. 8, 84-85 (1984)

[Ps] Palais, R. S.: Morse theory on Hilbert manifolds. Topology 2, 299-340 (1963)

[Pu] Pansu, P.: Sur l'article de M. Gromov, Preprint, Ecole Polytechnique, Palaiseau 1986

[P] Poincaré, H.: Sur une theoreme de geometrie, Rend. Circolo Mat. Palermo 33, 375-407 (1912)

[Q] Qinn, F.: Transversal approximation on Banach manifolds. In: Proc. Symp. Pure Math. vol. 15. Providence, RI: AMS 1970

[Ra] Rabinowitz, P. H.: Periodic solutions of Hamiltonian systems. Commun. Pure Appl. Math. 31, 336-352 (1979)

[Ry] Rybakowski, K. P.: The homotopy index and partial differential equations, Universitext. Berlin, Heidelberg, New York: Springer 1987

[RZ] Rybakowski, K. P., Zehnder, E.: A Morse equation in Conley's index theory for semiflows on metric spaces. Ergod. Theoret Dyn. Sys. 5, 123-143 (1985)

[SU] Sachs, J., Uhlenbeck, K. K.: The existence of minimal 2-spheres. Ann. Math. 113, 1-24 (1981)

[S1] Sikorav, J. C.: Points fixes d'un symplectomorphisme homologue a l'identite. J. Diff. Geom. 22, 49-79 (1982)

[S2] — : Homologie de Novikov associee a une classe de cohomologie reelle de degree un these, Orsay, 1987

[Si] Simon, C. P.: A bound for the fixed point index of an area preserving map with applications to mechanics. Invent. Math. 26, 187-200 (1974)

[Sm] Smale, S.: An infinite dimensional version of Sard's theorem. Am. J. Math. 87, 213-221 (1973)

[Sp] Spanier, E.: Algebraik topology. New York: McGraw-Hill 1966

[T1] Taubes, C. H.: Self-dual connections on manifolds with indefinite intersection matrix. J. Diff. Geom. 19, 517-5670 (1984)

[T2] —-: Gauge theory on asymptotically periodic 4-manifolds. J. Diff. Geom. 25, 363-430 (1987)

[V] Viterbo, C.: Intersections de sous-varietes lagrangiennes, fonctionelles d'action et indice des systemes hamiltoniens. Preprint 1986

[W1] Weinstein, A.: Periodic orbits for convex Hamiltonian systems. Ann. Math. 108, 507-518 (1978) 
[W2] — : On extending the Conley Zehnder fixed point theorem to other manifolds. Proc. Symp. Pure Math. vol. 45. Providence, R. J.: AMS 1986

[Wi] Witten, E.: Supersymmetry and Morse theory. J. Diff. Geom. 17, 661-692 (1982)

[Wo] Wolfson, J. G.: A P.D.E. proof of Gromov's compactness of pseudoholomorphic curves, preprint, Tulane University 1986

[Z] Zehnder, E.: Periodic solutions of Hamiltonian equations. In: Lecture Notes in Mathematics, vol. 1031. pp, 172-1213 Berlin, Heidelberg, New York: Springer 1983

Communicated by A. Jaffe

Received May 3, 1988; in revised from September 9, 1988 
